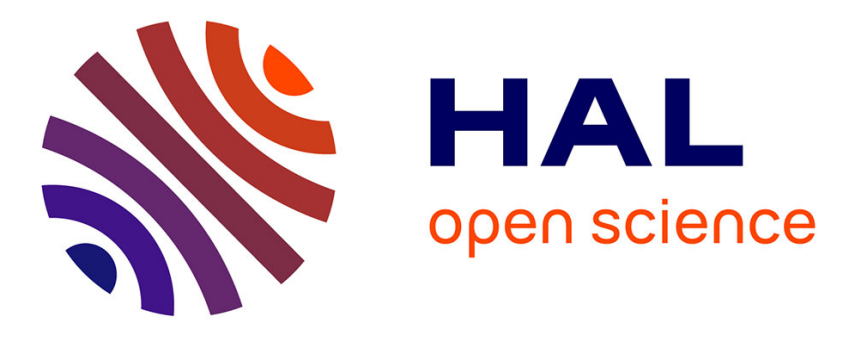

\title{
A fully integrated noise robust strategy for the identification of constitutive laws from digital images \\ Julien Réthoré
}

\section{To cite this version:}

Julien Réthoré. A fully integrated noise robust strategy for the identification of constitutive laws from digital images. International Journal for Numerical Methods in Engineering, 2010, 84, pp.631-660. 10.1002/nme.2908 . hal-00938286

\section{HAL Id: hal-00938286 \\ https://hal.science/hal-00938286}

Submitted on 27 May 2021

HAL is a multi-disciplinary open access archive for the deposit and dissemination of scientific research documents, whether they are published or not. The documents may come from teaching and research institutions in France or abroad, or from public or private research centers.
L'archive ouverte pluridisciplinaire HAL, est destinée au dépôt et à la diffusion de documents scientifiques de niveau recherche, publiés ou non, émanant des établissements d'enseignement et de recherche français ou étrangers, des laboratoires publics ou privés.

\section{(c)(1)}

Distributed under a Creative Commons Attribution| 4.0 International License 


\title{
A fully integrated noise robust strategy for the identification of constitutive laws from digital images
}

\author{
Julien Réthoré ${ }^{* \dagger}$ \\ Laboratoire de Mécanique des Contacts et des Structures (LaMCoS), INSA Lyon/Université de Lyon/UMR CNRS \\ 5259, 20 Avenue des Sciences, F-69621 Villeurbanne Cedex, France
}

Constitutive parameter identification has been greatly improved by the achievement of full-field measurements. In this context, noise sensitivity has been shown to be of great importance. It is crucial to incorporate noise sensitivity minimization in the design of robust identification procedures. In this paper, we investigate noise sensitivity reduction techniques for constitutive parameter identification based on Finite Element Model Updating. After examining the existing strategies, we propose a single step algorithm based on a mixed optical/mechanical cost function. The key point of this novel procedure is that no boundary conditions are needed. A first example on a real case illustrates the advantages of the proposed methodology in terms of noise sensitivity. A second example shows its capabilities to identify a non-linear consitutive law.

KEY WORDS: digital image correlation; constitutive law identification; noise sensitivity; integrated approach

\section{INTRODUCTION}

Constitutive parameter identification has been greatly improved by the achievement of full-field measurements, e.g. by digital image correlation (DIC). Many different techniques have been developed to extract consitutive parameters from a displacement field. These techniques can be sorted into two groups.

In the first group, the identification directly extracts the constitutive parameters from the measured displacement field. Based on various cost functions, such as constitutive equation gap, reciprocity gap, equilibrium gap (see Avril et al. [1] for an overview), the constitutive parameters are searched as the minimum of the cost function. The Virtual Field Method [2] can also be sorted in this group as the identification is directly performed from the measured strain field. Note that for

\footnotetext{
${ }^{*}$ Correspondence to: Julien Réthoré, Laboratoire de Mécanique des Contacts et des Structures (LaMCoS), INSA Lyon, 20 Avenue des Sciences, F-69621 Villeurbanne Cedex, France.

${ }^{\dagger}$ E-mail: julien.rethore@insa-lyon.fr
} 
all these techniques except the Virtual Field Method, the constitutive parameters are formulated as a quadratic functional of the gradient of the measured displacement. This may lead to systematic bias and non-optimality in terms of noise sensitivity. To improve noise robustness, the identification problem can be reconditioned as proposed in Roux and Hild [3] for the equilibrium gap method. The Virtual Field Method is a linear form of the measured strain and thus does not exhibit systematic bias. The not so constrained virtual fields that are used to extract material properties can be specialized to minimize the noise sensitivity. The reader may refer to Avril and Pierron [4] for an in-depth analysis of the noise robustness of these techniques.

For the second group, named the Finite Element Model Updating (FEMU) [5] family, the idea is to update the parameters of a constitutive model so that the result of a numerical simulation, run under appropriate boundary conditions, matches as fairly as possible the experimental field in the sense of a given norm (or cost function). Initially formulated using a gap on the global data from the testing machine [5], the use of full-field measurement techniques allows to reformulate the updating cost function as a gap between the experimental and simulated displacement or strain (see for example Lecompte et al. [6] or Genovese et al. [7]). To minimize the gap between experimental and simulated fields, a Levenberg-Marquardt procedure [8] is often used but genetic algorithms or Bayesian techniques can also be implemented [9]. Considering noise sensitivity, FEMU leads to the minimization of the norm of the gap between measured and computed displacements (or strains) with respect to constitutive parameters. With this approach, no systematic bias is obtained and optimal noise sensitivity should be reached as we will show in the sequel.

Dealing with experimental data, it is crucial to incorporate noise sensitivity minimization in the design of identification or extraction techniques to improve their robustness. One way to minimize noise sensitivity is to make the identification procedure as direct as possible. The measurement and the numerical simulation should use the same basis functions. Indeed, usually performed on independent zone of interests [10], the experimental displacement field cannot be compared directly to a displacement field computed by a finite element analysis. The measured displacement field must be projected onto the finite element basis (the one used for the numerical simulations) so that it can be compared to the numerical simulation results. This projection step may have an influence on the identification result as it modifies the measured displacement. To avoid this projection, DIC may be performed using the same basis function as the mechanical simulation. In this spirit, Besnard et al. [11] and Réthoré et al. [12,13] give representative examples of the use of base functions that are widely used for numerical simulations (FEM, X-FEM, Non-Uniform Rational BSpline (NURBS),...) in DIC. Another route is to supplement the minimization of the cost function with a noise robustness condition. Grédiac et al. [2], in the context of the Virtual Field Method, or Réthoré et al. [14], for the extraction of stress intensity factors, have shown how noise sensitivity minimization can be included in the extraction technique. As an alternative, mechanical equations (balance of momentum) can be introduced at the earliest stage of the identification procedure. Introducing the balance of momentum equations into the DIC algorithm, as in Réthore et al. [15] for linear elasticity, acts as a filter removing the unphysical components of the displacement and consequently removes noise.

We start by investigating the perturbative behavior of identification procedures within the family of the FEMU technique. Then we suggest and validate solutions that decrease noise sensitivity. We propose a fully coupled experimental/numerical procedure that is optimal in terms of noise sensitivity and mesh dependence. The first section briefly describes how DIC is formulated in a global manner so that finite element shape functions can be used. The following section is dedicated to FEMU, regularized and optimal approaches, their noise sensitivity and their application to the 
identification of the Poisson's ratio of a steel plate. As the strain levels in the elastic regime are very low for steel, this application allows to classify the analyzed techniques in terms of robustness. After analyzing the results, a fully coupled method is proposed that circumvents the drawbacks of the other approaches. After the first example is re-analyzed with the proposed strategy, a second example illustrates its capability to identify a damage law for a composite material.

\section{DIGITAL IMAGE CORRELATION}

From two gray level images $f$ and $g$ of a given sample, DIC consists of a non-linear minimization process that identifies the displacement field $\boldsymbol{u}$ that produces the advection of the local texture:

$$
\boldsymbol{u}=\operatorname{Arg} \operatorname{Min} \sum_{p \in \operatorname{ROI}}\left[f\left(\mathbf{x}_{\mathbf{p}}\right)-g\left(\mathbf{x}_{\mathbf{p}}+\mathbf{u}\left(\mathbf{x}_{\mathbf{p}}\right)\right)\right]^{2},
$$

where $p$ is the index of a pixel within the Region Of Interest (ROI) over which the computation is performed and $\mathbf{x}_{\mathbf{p}}$ the co-ordinates of pixel $p$ on the image frame. Following Besnard et al. [11], we adopt a Finite Element discretization for the displacement field. The numerical simulation and the displacement measurement can be performed using the same basis functions and no projection is required during the identification procedure. The discretized displacement field reads as:

$$
\boldsymbol{u}(\mathbf{x})=\sum_{k \in \mathscr{N}} u_{k} N_{k}(\mathbf{x})
$$

where $\mathscr{N}$ is the set of finite element degrees of freedom (DOF), and $\boldsymbol{N}_{k}$ the finite element shape functions. From a first guess for the displacement, an iterative procedure is run until convergence is reached. Assuming that the solution increment du is small enough, a first-order Taylor expansion of $g\left(\mathbf{x}_{\mathbf{p}}+\mathbf{u}\left(\mathbf{x}_{\mathbf{p}}\right)+\mathrm{d} \mathbf{u}\left(\mathbf{x}_{\mathbf{p}}\right)\right)$ is used to linearize the functional. After some manipulations, we obtain that the solution increment is calculated through:

$$
\left[\tilde{\mathbf{M}}_{\mathrm{DIC}}\right]\{\mathrm{d} \mathbf{U}\}=\left\{\tilde{\mathbf{b}}_{\mathrm{DIC}}\right\},
$$

with

$$
\left[\tilde{\mathbf{M}}_{\mathrm{DIC}}\right]=[\mathbf{N}]^{\mathrm{T}}[\nabla \mathbf{G}][\nabla \mathbf{G}][\mathbf{N}]
$$

and

$$
\left\{\tilde{\mathbf{b}}_{\mathrm{DIC}}\right\}=[\mathbf{N}]^{\mathrm{T}}[\nabla \mathbf{G}]\{\mathbf{F}-\mathbf{G}\},
$$

$\nabla$ denoting spatial derivation. $[\mathbf{N}]$ is a matrix that collects the value of the basis functions at the pixels, $[\nabla \mathbf{G}]$ a diagonal matrix that collects the values of $\nabla g\left(\mathbf{x}_{\mathbf{p}}+\mathbf{u}\left(\mathbf{x}_{\mathbf{p}}\right)\right)$ and $\{\mathbf{F}-\mathbf{G}\}$ a vector containing the values of $f\left(\mathbf{x}_{\mathbf{p}}\right)-g\left(\mathbf{x}_{\mathbf{p}}+\mathbf{u}\left(\mathbf{x}_{\mathbf{p}}\right)\right)$. For the sake of clarity, indices indicating the iteration number have been omitted. For a detailed description of the algorithm in a multiscale framework and its implementation the interested reader may refer to Réthoré et al. [16]. To reduce the computational cost, a modified algorithm is adopted: we substitute $\nabla \mathbf{G}$, which depends on the current solution vector, by $\nabla \mathbf{F}$. The modified system to solve thus becomes

$$
\left[\mathbf{M}_{\mathrm{DIC}}\right]\{\mathrm{d} \mathbf{U}\}=\left\{\mathbf{b}_{\mathrm{DIC}}\right\} \text {, }
$$

with

$$
\left[\mathbf{M}_{\text {DIC }}\right]=[\mathbf{N}]^{\mathrm{T}}[\nabla \mathbf{F}][\nabla \mathbf{F}][\mathbf{N}],
$$


and

$$
\left\{\mathbf{b}_{\text {DIC }}\right\}=[\mathbf{N}]^{\mathrm{T}}[\nabla \mathbf{F}]\{\mathbf{F}-\mathbf{G}\} .
$$

The advantage of such a modification is that $\mathbf{M}_{\text {DIC }}$ and parts of $\mathbf{b}_{\text {DIC }}$ can be computed once and for all. Note that when convergence is reached, $\nabla \mathbf{F}$ equals $\nabla \mathbf{G}$. After a few iterations (2 or 3), the modified algorithm and the initial one reveal close to identical.

Following Réthore et al. [17], we can derive the correlation kernel of the noise affecting the displacement due to a spatially uncorrelated image noise. Instead of considering that both reference and deformed images are affected by noise (of standard deviation $\sigma$ ), it is more convenient to report the noise on the deformed image only and to increase its amplitude by a factor 2 . We have

$$
\langle\{\boldsymbol{\delta} \mathbf{G}\}\rangle=0, \quad\left\langle\{\boldsymbol{\delta} \mathbf{G}\}\{\boldsymbol{\delta} \mathbf{G}\}^{\mathrm{T}}\right\rangle=2[\mathbf{I}] \sigma^{2},
$$

where $\boldsymbol{\delta} \mathbf{G}$ is the vector collecting the values of the realizations of the deformed image noise, $\langle\bullet\rangle$ is the average operator over the realizations of $\bullet, \sigma$ is the standard deviation of the image noise, and $\mathbf{I}$ the identity matrix whose dimension is the number of pixels in the ROI. In Equation (6), only $\mathbf{G}$ is noisy. The displacement perturbation thus writes as

$$
\{\boldsymbol{\delta} \mathbf{U}\}=\left[\mathbf{M}_{\mathrm{DIC}}\right]^{-1}[\mathbf{N}]^{\mathrm{T}}[\nabla \mathbf{F}]\{\boldsymbol{\delta} \mathbf{G}\} .
$$

From this equation, we obtain that the displacement perturbation $\boldsymbol{\delta} \boldsymbol{U}$ has no systematic bias

$$
\langle\{\boldsymbol{\delta} \mathbf{U}\}\rangle=0,
$$

and that its covariance matrix is written as follows:

$$
\left\langle\{\boldsymbol{\delta} \mathbf{U}\}\{\boldsymbol{\delta} \mathbf{U}\}^{\mathrm{T}}\right\rangle=2\left[\mathbf{M}_{\mathrm{DIC}}\right]^{-1}\left[\mathbf{M}_{\mathrm{DIC}}\right]\left[\mathbf{M}_{\mathrm{DIC}}\right]^{-1} \sigma^{2}=2\left[\mathbf{M}_{\mathrm{DIC}}\right]^{-1} \sigma^{2} .
$$

The noise affecting the displacement is thus not a white noise. Its correlation kernel is given by $\left[\mathbf{M}_{\text {DIC }}\right]^{-1}$. The displacement noise is not uniform and depends on the texture of the image around each node of the finite element mesh. $\left[\mathbf{M}_{\mathrm{DIC}}\right]^{-1}$ is not diagonal which leads to a spatially correlated noise. In practice, $\left[\mathbf{M}_{\mathrm{DIC}}\right]^{-1}$ can be seen as a confidence indicator on the estimation of each DOF.

\section{EXISTING FEMU-BASED PROCEDURES}

In this section, the existing FEMU procedures are formulated. Figure 1 shows a generic algorithm of FEMU. Many variants can be formulated depending on the cost function $\Phi$ (global forces, displacement, mixed displacement and forces, strains,...) and the updating of the parameters (Gauss-Newton, Levenberg-Marquardt, genetic algorithm, Bayesian procedure,...). However, the principle remains identical, that is updating the parameters of a finite element model so that the minimum of a cost function is reached. This leads to a two-steps procedure: first to extract the boundary conditions to apply on the finite element model and/or the measured displacement and second to adjust the material parameters to minimize the cost function. A formalism is proposed to study the propagation of the image noise through the displacement measurement and the constitutive parameters identification. 


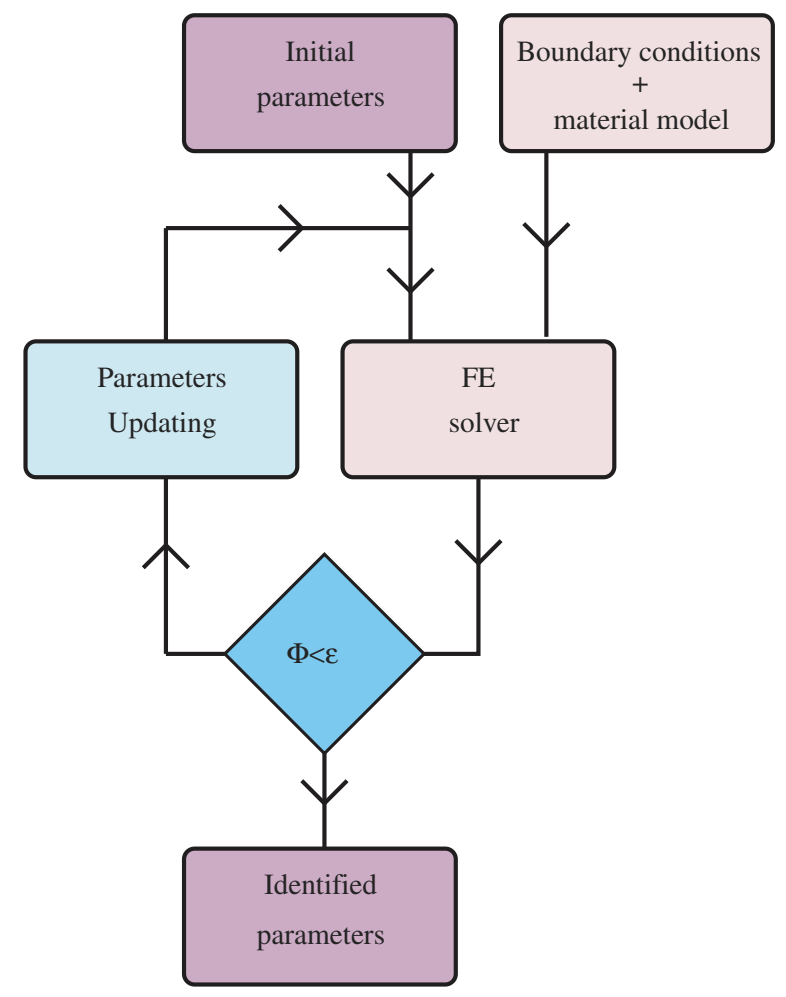

Figure 1. Finite Element Model Updating generic algorithm.

\subsection{Finite Element Model Updating}

FEMU is a non-linear minimization problem whose goal is to identify the parameters of a given consitutive model from a given displacement field. We define $\boldsymbol{\Lambda}$ as a vector that contains the parameters of the constitutive model. We concentrate herein on a displacement gap formulation. Its evaluation is obtained by:

$$
\boldsymbol{\Lambda}=\operatorname{Arg} \operatorname{Min}[\mathbf{U}-\mathbf{V}(\boldsymbol{\Lambda})]^{\mathrm{T}}[\mathbf{U}-\mathbf{V}(\boldsymbol{\Lambda})],
$$

where $\mathbf{V}$ is the solution vector of a mechanical simulation run with the constitutive parameters $\boldsymbol{\Lambda}$ and the appropriate boundary conditions. An increment $\mathrm{d} \boldsymbol{\Lambda}$ of the constitutive parameters is obtained by solving the following system:

$$
\left[\mathbf{M}_{\text {FEMU }}\right]\{\mathrm{d} \boldsymbol{\Lambda}\}=\left\{\mathbf{b}_{\text {FEMU }}\right\},
$$

with

$$
\left[\mathbf{M}_{\mathrm{FEMU}}\right]=\left[\frac{\Delta \mathbf{V}}{\Delta \mathbf{\Lambda}}\right]^{\mathrm{T}}\left[\frac{\Delta \mathbf{V}}{\Delta \mathbf{\Lambda}}\right],
$$

and

$$
\left\{\mathbf{b}_{\text {FEMU }}\right\}=\left[\frac{\Delta \mathbf{V}}{\Delta \boldsymbol{\Lambda}}\right]^{\mathrm{T}}\{\mathbf{U}-\mathbf{V}(\boldsymbol{\Lambda})\},
$$

where $\Delta \mathbf{V} / \Delta \Lambda$ collects the variation of the simulated mechanical solution $\mathbf{V}$ with respect to the constitutive parameters $\Lambda$ (computed by finite differences for example). 
The same perturbation analysis can be carried out as in the case of DIC (Equation (12)). Assuming $\mathbf{V}$ has no noise, we obtain:

$$
\left\langle\{\boldsymbol{\delta} \boldsymbol{\Lambda}\}\{\boldsymbol{\delta} \boldsymbol{\Lambda}\}^{\mathrm{T}}\right\rangle=\left[\mathbf{M}_{\mathrm{FEMU}}\right]^{-1}\left[\frac{\Delta \mathbf{V}}{\Delta \boldsymbol{\Lambda}}\right]\left\langle\{\boldsymbol{\delta} \mathbf{U}\}\{\boldsymbol{\delta} \mathbf{U}\}^{\mathrm{T}}\right\rangle\left[\frac{\Delta \mathbf{V}}{\Delta \boldsymbol{\Lambda}}\right]^{\mathrm{T}}\left[\mathbf{M}_{\mathrm{FEMU}}\right]^{-1} .
$$

Using Equation (12), the following form is obtained:

$$
\left\langle\{\boldsymbol{\delta} \boldsymbol{\Lambda}\}\{\boldsymbol{\delta} \boldsymbol{\Lambda}\}^{\mathrm{T}}\right\rangle=2\left[\mathbf{M}_{\mathrm{FEMU}}\right]^{-1}\left[\frac{\Delta \mathbf{V}}{\Delta \boldsymbol{\Lambda}}\right]\left[\mathbf{M}_{\mathrm{DIC}}\right]^{-1}\left[\frac{\Delta \mathbf{V}}{\Delta \boldsymbol{\Lambda}}\right]^{\mathrm{T}}\left[\mathbf{M}_{\mathrm{FEMU}}\right]^{-1} \sigma^{2} .
$$

\subsection{Regularized Finite Element Model Updating (FEMU-R)}

Leclerc et al. [18] recently introduced a change in the scalar product of Equation (13) to balance the contribution of each finite element DOF with respect to its sensitivity level given by $\left[\mathbf{M}_{\mathrm{DIC}}\right]$. With this regularization, the cost function reads as

$$
\boldsymbol{\Lambda}=\operatorname{Arg} \operatorname{Min}[\mathbf{U}-\mathbf{V}(\boldsymbol{\Lambda})]^{\mathrm{T}}\left[\mathbf{M}_{\mathrm{DIC}}\right][\mathbf{U}-\mathbf{V}(\boldsymbol{\Lambda})] .
$$

The system to solve is derived from the above equation:

$$
\left[\mathbf{M}_{\text {FEMU-R }}\right]=\left[\frac{\Delta \mathbf{V}}{\Delta \mathbf{\Lambda}}\right]^{\mathrm{T}}\left[\mathbf{M}_{\mathrm{DIC}}\right]\left[\frac{\Delta \mathbf{V}}{\Delta \mathbf{\Lambda}}\right]
$$

and

$$
\left\{\mathbf{b}_{\mathrm{FEMU}-\mathrm{R}}\right\}=\left[\frac{\Delta \mathbf{V}}{\Delta \boldsymbol{\Lambda}}\right]^{\mathrm{T}}\left[\mathbf{M}_{\mathrm{DIC}}\right]\{\mathbf{U}-\mathbf{V}(\boldsymbol{\Lambda})\} .
$$

The sensitivity analysis can be carried out in a similar manner. We get:

$$
\left\langle\{\boldsymbol{\delta} \boldsymbol{\Lambda}\}\{\boldsymbol{\delta} \boldsymbol{\Lambda}\}^{\mathrm{T}}\right\rangle=2\left[\mathbf{M}_{\text {FEMU-R }}\right]^{-1}\left[\frac{\Delta \mathbf{V}}{\Delta \boldsymbol{\Lambda}}\right]\left[\mathbf{M}_{\text {DIC }}\right]\left[\frac{\Delta \mathbf{V}}{\Delta \boldsymbol{\Lambda}}\right]^{\mathrm{T}}\left[\mathbf{M}_{\text {FEMU-R }}\right]^{-1} \sigma^{2} .
$$

Accounting for the expression of $\left[\mathbf{M}_{\mathrm{FEMU}-\mathrm{R}}\right]$ (Equation (20)) the uncertainty becomes:

$$
\left\langle\{\boldsymbol{\delta} \boldsymbol{\Lambda}\}\{\boldsymbol{\delta} \boldsymbol{\Lambda}\}^{\mathrm{T}}\right\rangle=2\left[\mathbf{M}_{\text {FEMU-R }}\right]^{-1} \sigma^{2} .
$$

As the contribution of each DOF has been weighted with respect to its level of confidence, this approach is supposed to reduce the noise sensitivity of the identification process. As we will show later, it allows to obtain optimal robustness.

\subsection{Mechanical image correlation (MIC)}

An identification procedure should be as direct as possible to reduce noise sensitivity. For this purpose, we suggest to reformulate the procedure described in Section 3.2. The idea is to try and minimize the mismatching of the images directly from the consitutive parameters. The cost function becomes:

$$
\boldsymbol{\Lambda}=\operatorname{Arg} \operatorname{Min} \sum_{p \in \operatorname{ROI}}\left[f\left(\mathbf{x}_{\mathbf{p}}\right)-g\left(\mathbf{x}_{\mathbf{p}}+\mathbf{v}\left(\mathbf{x}_{\mathbf{p}}\right)\right)\right]^{2} .
$$


In this strategy, the deformed image $g$ is corrected by $\mathbf{v}$ which is the simulated displacement using the current estimation of the constitutive parameters and appropriate boundary conditions. Using contracted notations, the minimization is achieved following the same path as for DIC or FEMU. We obtain the solution increment from:

$$
\left[\mathbf{M}_{\mathrm{MIC}}\right]\{\mathrm{d} \boldsymbol{\Lambda}\}=\left\{\mathbf{b}_{\mathrm{MIC}}\right\},
$$

with

$$
\left[\mathbf{M}_{\mathrm{MIC}}\right]=\left[\frac{\Delta \mathbf{G}}{\Delta \mathbf{\Lambda}}\right]^{\mathrm{T}}\left[\frac{\Delta \mathbf{G}}{\Delta \mathbf{\Lambda}}\right] .
$$

$\Delta \mathbf{G}$ is linearized and written as $[\nabla \mathbf{G}][\mathbf{N}] \Delta \mathbf{V}$. The matrix $\left[\mathbf{M}_{\mathrm{MIC}}\right]$ finally recasts in the following form:

$$
\left[\mathbf{M}_{\mathrm{MIC}}\right]=\left[\frac{\Delta \mathbf{V}}{\Delta \mathbf{\Lambda}}\right]^{\mathrm{T}}[\mathbf{N}]^{\mathrm{T}}[\nabla \mathbf{G}][\nabla \mathbf{G}][\mathbf{N}]\left[\frac{\Delta \mathbf{V}}{\Delta \mathbf{\Lambda}}\right]=\left[\frac{\Delta \mathbf{V}}{\Delta \mathbf{\Lambda}}\right]^{\mathrm{T}}\left[\mathbf{M}_{\mathrm{DIC}}\right]\left[\frac{\Delta \mathbf{V}}{\Delta \mathbf{\Lambda}}\right],
$$

and vector $\left\{\mathbf{b}_{\mathrm{MIC}}\right\}$ as:

$$
\left\{\mathbf{b}_{\mathrm{MIC}}\right\}=\left[\frac{\Delta \mathbf{V}}{\Delta \boldsymbol{\Lambda}}\right]^{\mathrm{T}}[\mathbf{N}]^{\mathrm{T}}[\nabla \mathbf{F}]\{\mathbf{F}-\mathbf{G}(\boldsymbol{\Lambda})\},
$$

when the same modification as for DIC is adopted, i.e. $\nabla \mathbf{G}$ is substituted by $\nabla \mathbf{F}$. Solving this system, the constitutive parameters increment is directly obtained from the minimization of the mismatching between the two images. Note that, Leclerc et al. [18] proposed a so-called 'integrated' approach. It consists in using $[\mathbf{N}][\Delta \mathbf{V} / \Delta \mathbf{\Lambda}]$ as a basis function for DIC so that $\mathrm{d} \boldsymbol{\Lambda}$ is also directly obtained by achieving the DIC minimization. The system to solve is exactly the same as the proposed MIC approach, which makes these two approaches identical. They only differ in the way the problem is formulated.

If we derive the influence of noise for MIC, the following result is obtained:

$$
\left\langle\{\boldsymbol{\delta} \boldsymbol{\Lambda}\}\{\boldsymbol{\delta} \boldsymbol{\Lambda}\}^{\mathrm{T}}\right\rangle=2\left[\mathbf{M}_{\mathrm{MIC}}\right]^{-1} \sigma^{2} .
$$

An interesting result is that matrices $\left[\mathbf{M}_{\mathrm{MIC}}\right]$ and $\left[\mathbf{M}_{\mathrm{FEMU}-\mathrm{R}}\right]$ have the same expressions. As a consequence, the analysis proposed herein proves that the regularized two-steps procedure (FEMU-R) and the MIC approach (or 'integrated') have formally the same sensitivity to noise. The only difference is that the right-hand side of the system to solve involves a displacement residual for the FEMU-R procedure, whereas a gray level residual is invoked in the MIC strategy. In practice, the two solutions may also differ because of the matrix $[\Delta \mathbf{V} / \Delta \mathbf{\Lambda}]$ that is re-computed at each iteration. The identified set of parameters will also depend on the successive estimations of the $\mathbf{V}$ field. This will be illustrated in the following paragraph. In the present analysis, we study the propagation of the image noise through the identification algorithm and the field $\mathbf{V}$ is supposed to be noise free. Avril and Pierron [4] give complementary results as they reported that the maximum likelihood solution can only be obtained with a displacement gap-based FEMU if the boundary conditions are noise free. If not, they show a way to analyze the influence of noise in the field $\mathbf{V}$.

\subsection{Identification of a steel plate's Poisson's ratio}

A tensile test on a $15 \mathrm{~mm} \times 1.2 \mathrm{~mm}$ steel specimen is analyzed. The images are presented in Figure 2 . The pixel size is $75 \mu \mathrm{m}$ and the analyzed region is a rectangle of $800 \times 200$ pixels. Images have 


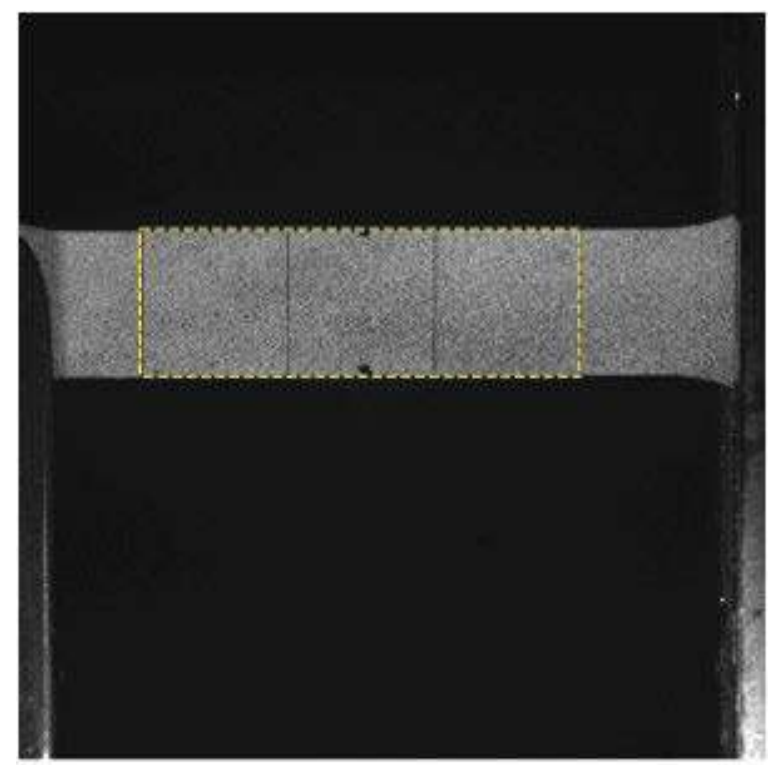

(a)

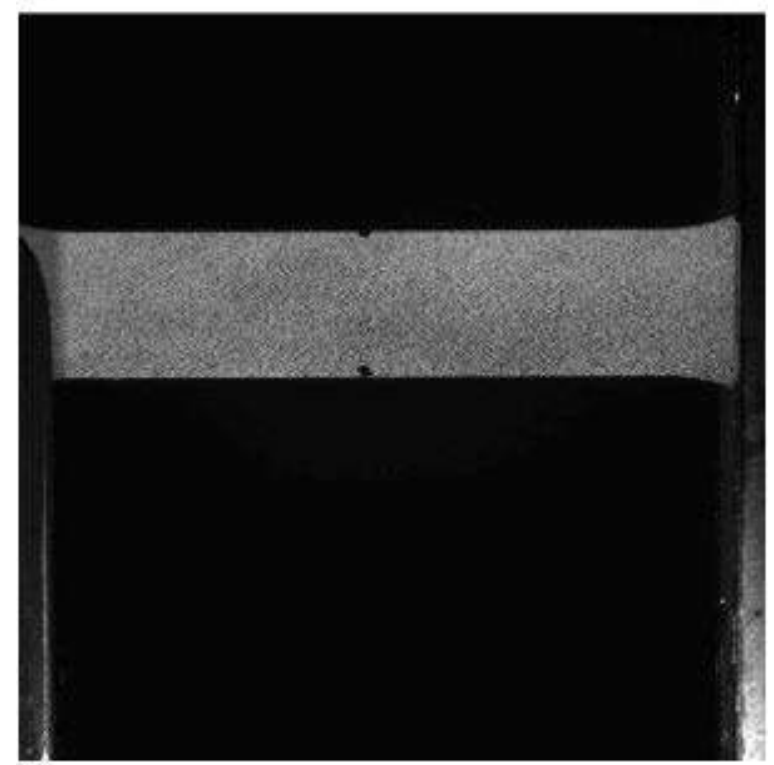

(b)

Figure 2. Initial and deformed image of the steel sample under tension. The finite element mesh with 200-pixel elements is visible on the initial image.

8-bit digitization. The aim of this example is to identify the Poisson's ratio $v$. As strains are very small for steel in the elastic regime (about $10^{-4}$ ), this problem is known to reach the limit resolution of DIC.

Figure 3(a) shows the deformed mesh resulting from a DIC analysis using 200-pixel piecewise linear elements. The amplification factor is 500 which shows that the strains are really small. Taking the average value for the longitudinal strain (see Figure 3(c)) $E_{x x}=0.04 \%$, and for the transverse strain $E_{y y}=-0.01 \%$, we obtain a value of 0.4 for $v$ which is a little higher than the usual one for such material. Taking the averaged strains to compute $v$ is the crudest filtering that one could apply. This is also the most appropriate if the test was uniaxial. The warped shapes 


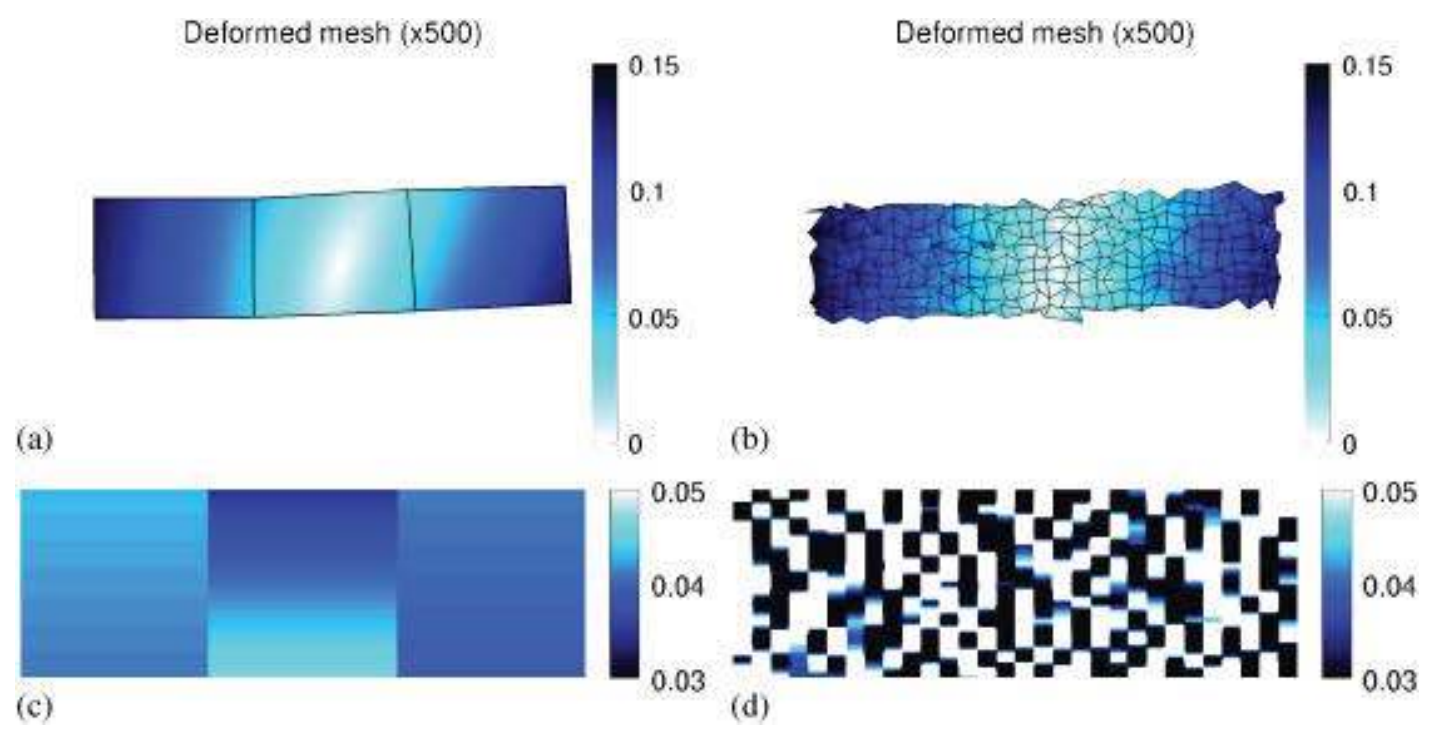

Figure 3. Displacement norm in pixel on the deformed mesh (a),(b) $(\times 500$ amplification) and longitudinal strain field (c),(d) (in per cent) for a DIC analysis with 200-pixel elements (a),(c) and a DIC analysis with 20-pixel elements (b),(d).

of the specimen in Figure 3(a),(b) show that bending that occurs during the test which cannot be considered as uniaxial. Consequently, Poisson's ratio cannot be identified by just averaging the strains. If a 20-pixel element analysis (see Figure 3(b)) is run, the longitudinal strain field seems to contain only noise as it can be seen in Figure 3(d) for which the color scale was kept unchanged. Taking the strain mean values, we obtain $v=0.6$. This confirms that the strain level is close to the limit resolution of DIC and that the test must not be considered as uniaxial.

First, the identification of Poisson's ratio is performed when Dirichlet boundary conditions coming from a 200-pixel elements DIC analysis are applied on the left and right edges of the mechanical simulations. Figure 4 shows the deformed mesh and the longitudinal strain field after $v$ has been identified using MIC on a 10 pixel element mesh. It is confirmed that the strains are really low and that the test was anything but a pure tensile test which is also in favor of an analysis using full-field measurements. In Figure 5, identified Poisson's ratios are plotted with error bars that correspond to an image noise $\sigma$ of 2 gray levels for 8-bit digitization. The results are in good agreement in the uncertainty range. FEMU shows the larger variations with the mesh size whereas MIC leads to mesh independence. For a 10-pixel elements and an image noise $\sigma$ of 2 gray levels, we obtain $0.264 \pm 0.017$ for FEMU, $0.3 \pm 0.013$ for FEMUR, and $0.283 \pm 0.015$ for MIC. For this element size, FEMU leads to a relative gap in the correlation displacement field of $8.31 \%$ whereas FEMU-R gives $4.56 \%$. If the mismatching of the images is calculated using the $\mathbf{V}$ field computed with the identified value of $v$, one obtains $0.88 \%$ of the image dynamic range for the two methods. For MIC, the same value of $0.88 \%$ of the image dynamic range is also obtained. These results are in agreement, the small variations of the results by MIC increases the confidence in this approach.

The evolution of the sensitivity to noise that is presented in Figure 5(b) shows that the optimal identification strategies give lower uncertainties (from about 0.008/gray level for FEMU to 0.007/gray level). Comparing the results obtained by FEMU-R and MIC in Figure 5, the following interpretation arises: whereas the sensitivity of these methods have been shown to be formally identical, small differences appear in favor of FEMU-R. Again, the right-hand side appearing in 


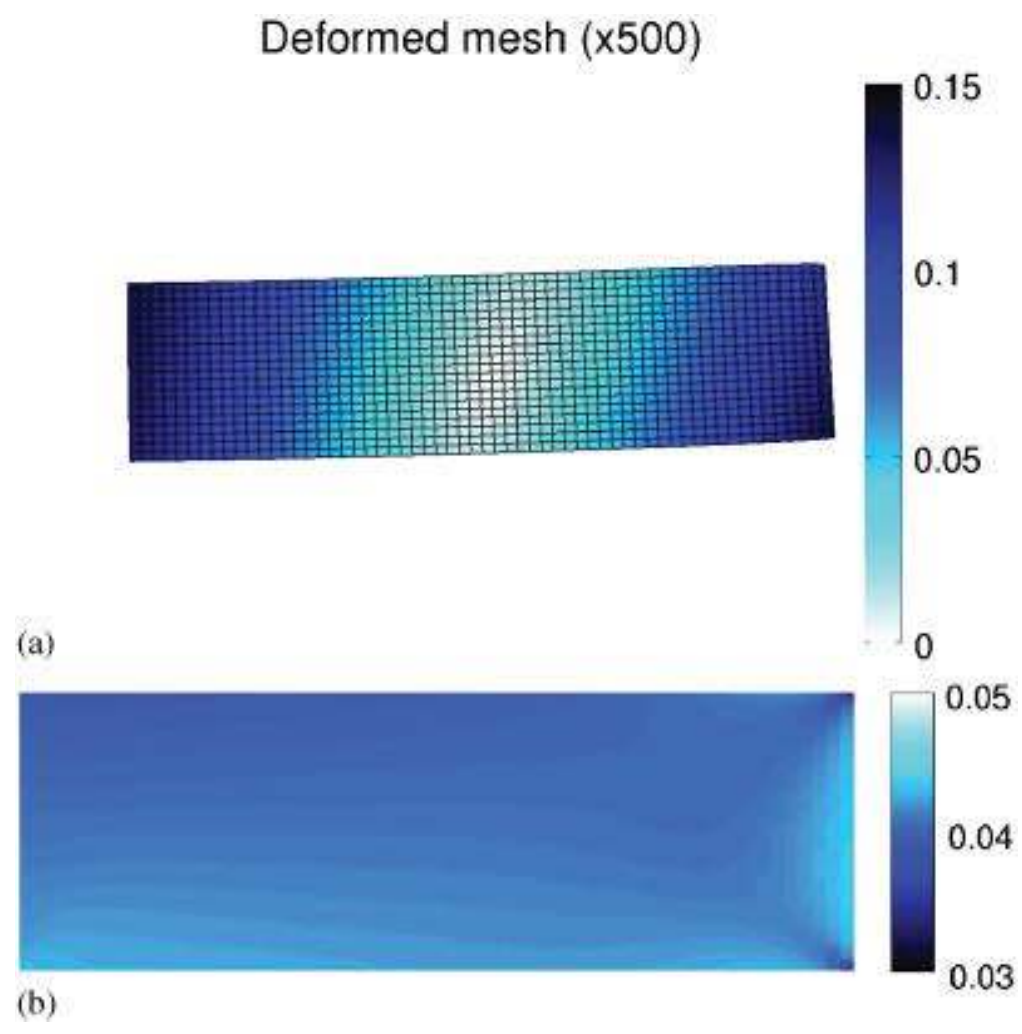

Figure 4. Displacement norm in pixel on the deformed mesh $(\times 500$ amplification $)$ and longitudinal strain field (in per cent) using 10-pixel elements for an MIC analysis with coarse mesh size boundary conditions.

the system of these methods being different, different results are obtained. Whereas FEMU-R leads to the lowest uncertainty, from Figure 5(a) it appears that the displacement-based residual (used in FEMU-R) does not allow to achieve the same invariance with the size of the element as obtained with a correlation-based residual (MIC). FEMU-R (and further FEMU) is based on the minimization of a displacement gap functional whereas MIC is based on the optical flow equation. The noise affecting the objective displacement (obtained from a pure DIC analysis) has its amplitude increased when the mesh size decreases and thus FEMU-R shows variations of the identified parameter with the mesh size. The noise in the images (and thus the noise source in MIC) remains unchanged when the mesh size decreases and MIC's results have less dependence on this parameter.

In Figure 6, we show the evolution of the identified $v$ and its noise sensitivity when the correlation analysis for the current element size is used as Dirichlet boundary conditions. This demonstrates that all the strategies exhibit a strong dependence on this parameter. Values for $v$ down to 0.05 are obtained with a sensitivity of 0.01 per gray level. One can also observe the influence of the boundary conditions for MIC in Figure 7. This figure shows the deformed mesh and the longitudinal strain field, respectively, for fine (10-pixel elements) boundary conditions. There are strong differences between the $\mathbf{V}$ fields (calculated with the converged $v$ ) with prescribed displacement coming from a fine DIC analysis or a coarse one (Figure 4). All the studied strategies prove very sensitive to this parameter.

The above results confirm that lower sensitivity to noise is obtained using FEMU-R and MIC as it was theoretically proved at the beginning of this section. Using low noise boundary conditions 


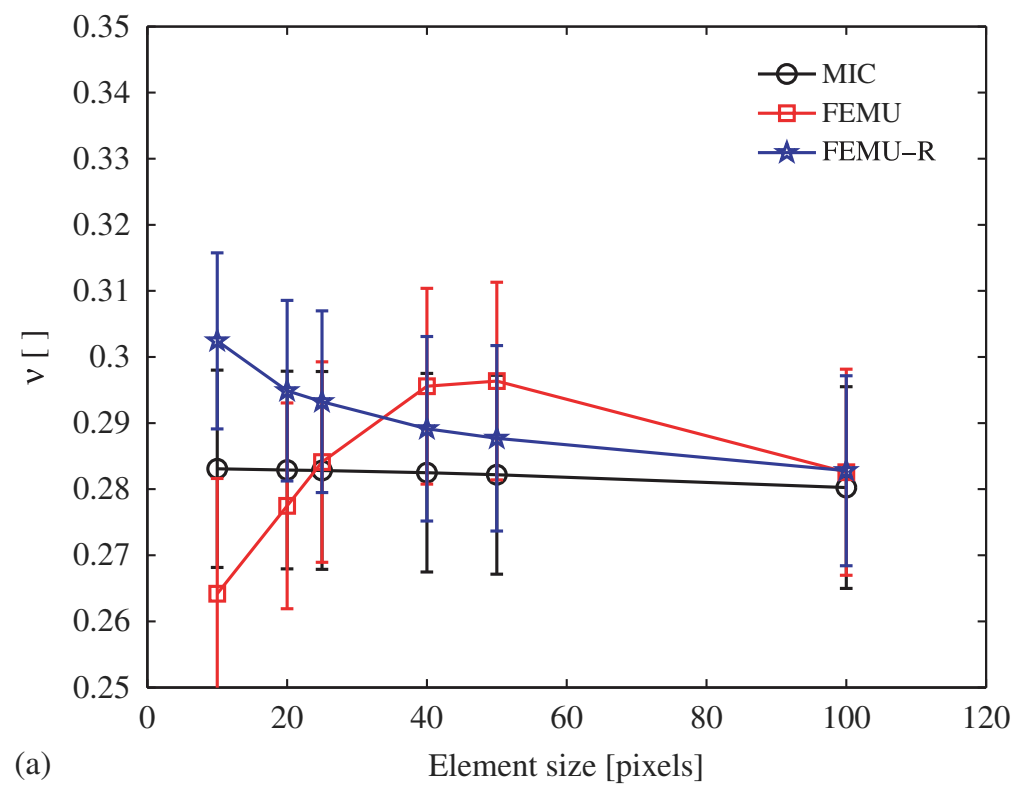

(a)

x $10^{-3}$

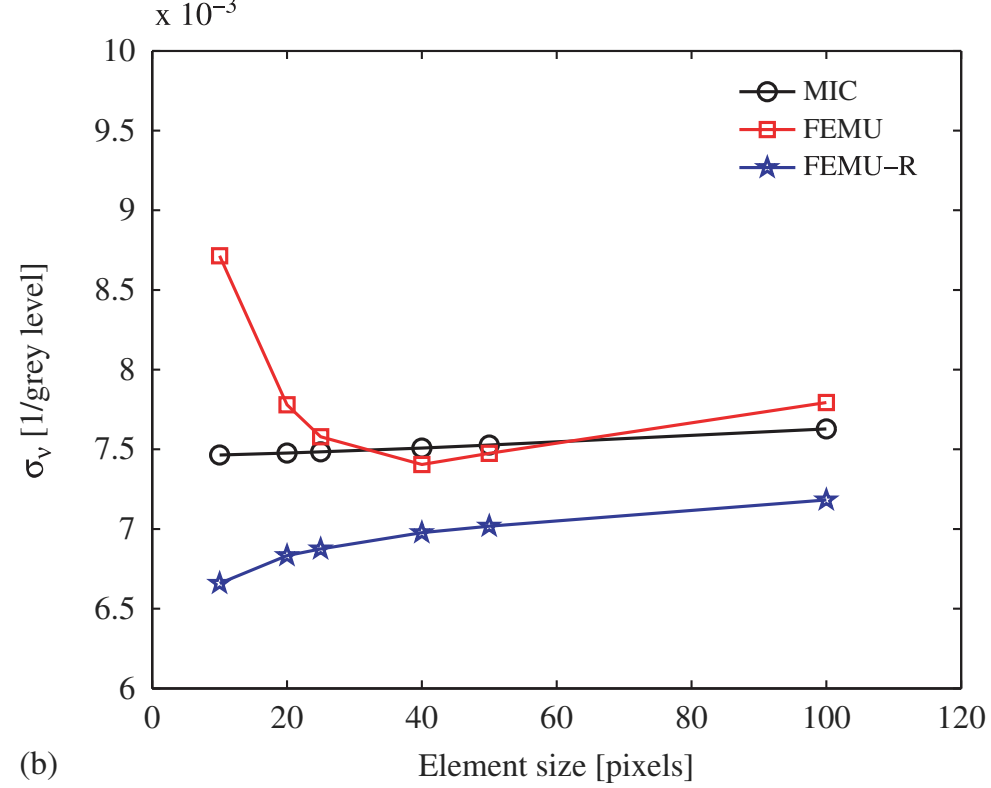

Figure 5. Evolution of the identified Poisson's ratio and its noise sensitivity as a function of the element size. The DIC analysis with 200-pixel elements is used as Dirichlet boundary conditions for the $\mathbf{V}$ field. Error bars correspond to an image noise of 2 gray levels.

(coming from a DIC analysis with 200-pixel elements) for calculating the $\mathbf{V}$ field, only the opticalbased formulation (MIC) allows for mesh independence. However, when noise affects the boundary conditions (i.e. using a DIC analysis with the current element size), all the strategies fail. This confirms the analysis of Avril and Pierron [4] who state that the maximum likelihood set of parameters can only be obtained if the boundary conditions used to obtain $\mathbf{V}$ are not affect by noise. To circumvent this drawback, a fully integrated strategy that does not require boundary conditions is proposed in the following section. 


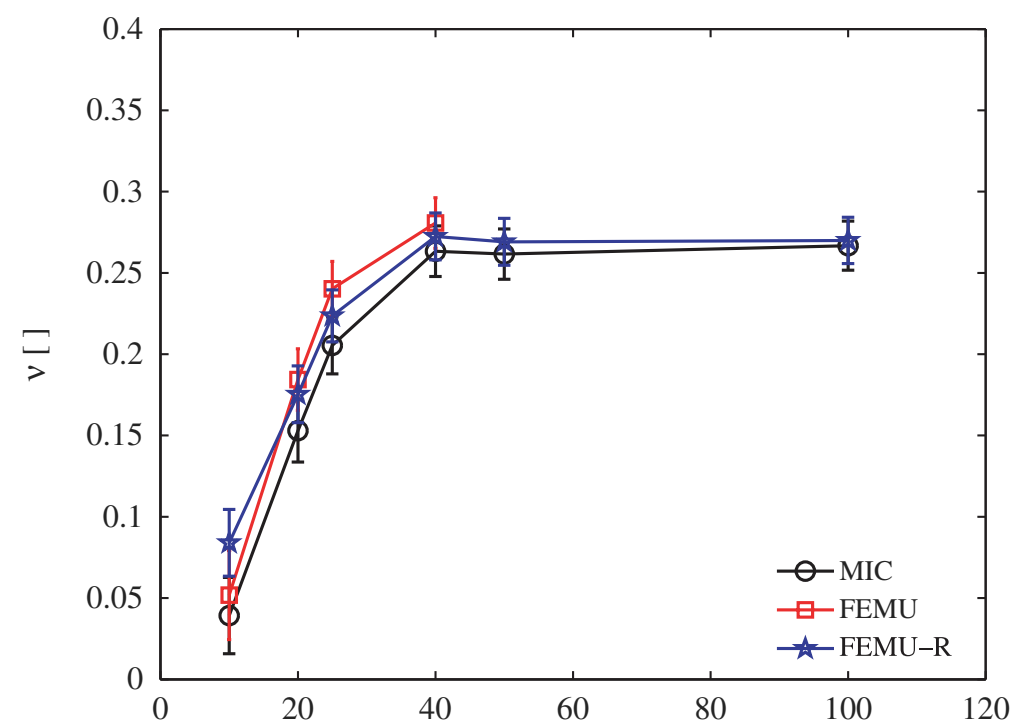

(a)

Element size [pixels]

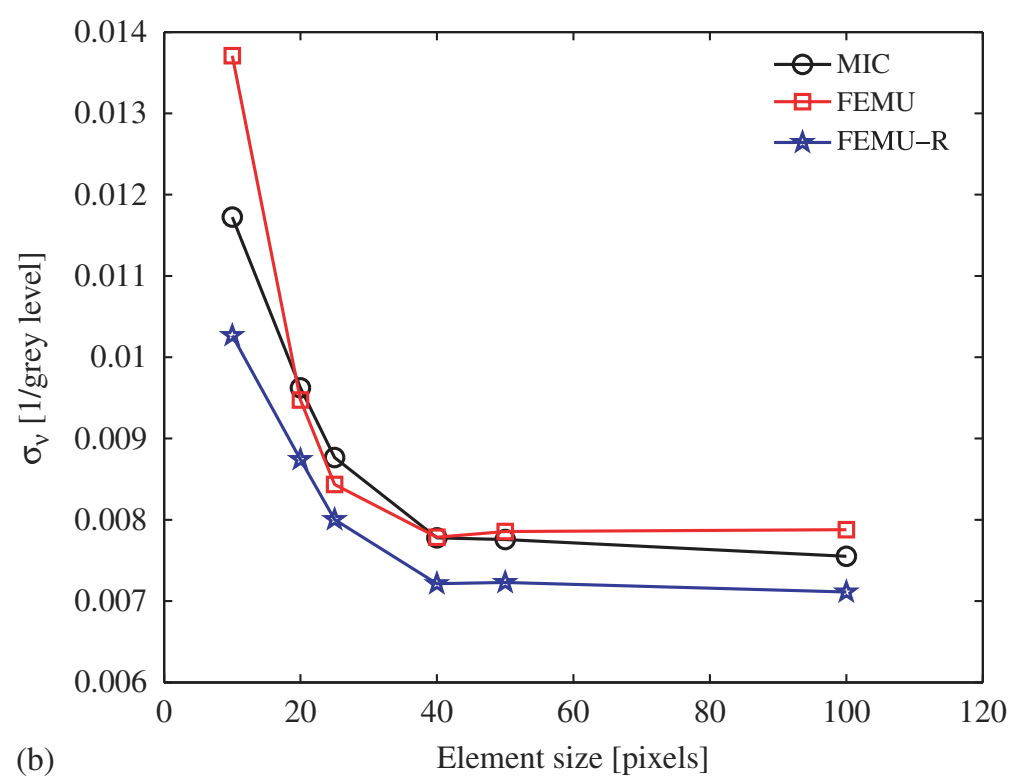

Figure 6. Evolution of the identified Poisson's ratio and its noise sensitivity as a function of the element size. The DIC analysis with the current element size is used as Dirichlet boundary conditions for the $\mathbf{V}$ field. Error bars correspond to an image noise of 2 gray levels.

\section{INTEGRATED MECHANICAL IMAGE CORRELATION (I-MIC)}

A common step for all the identification strategies described previously is the use of the $\mathbf{V}$ field. This field is obtained from a numerical simulation of a mechanical problem and therefore invokes boundary conditions. It was shown in the example that despite an optimal noise sensitivity, FEMU-R and MIC (and further FEMU), exhibit a strong dependence on these boundary conditions. In order to decrease this effect, we suggest to reformulate the above described two-steps procedures in a single step algorithm. It consists in extracting simultaneously from the images both the 

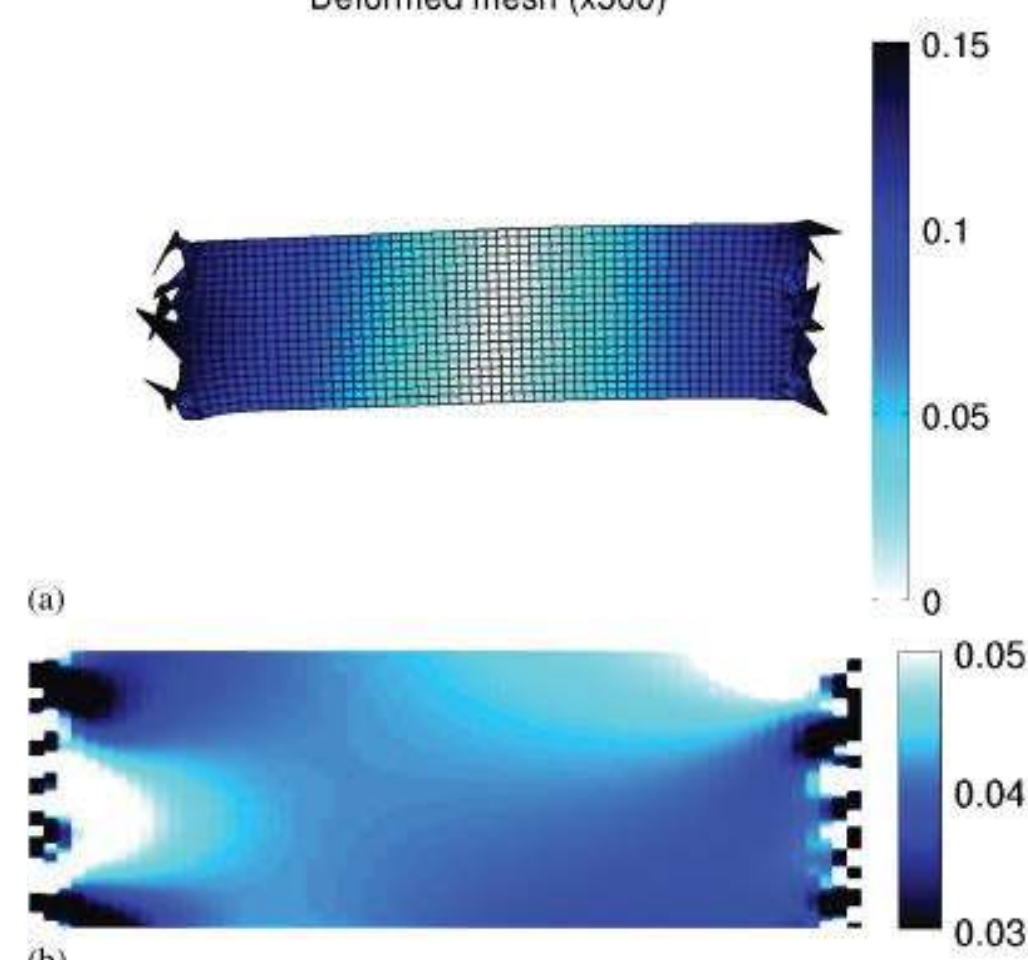

(b)

Figure 7. Displacement norm in pixel on the deformed mesh $(\times 500$ amplification $)$ and longitudinal strain field (in per cent) using 10-pixel elements for an MIC analysis with current mesh size boundary conditions.

displacement field and the constitutive parameters without requiring boundary conditions. For this purpose, a mechanically regularized optical cost function is adopted as proposed in Réthoré $e t$ al. [15] for DIC in linear elasticity. The cost function reads as:

$$
\{\boldsymbol{U}, \boldsymbol{\Lambda}\}=\operatorname{Arg} \operatorname{Min}\left(\frac{1}{m_{o}}\{\mathbf{F}-\mathbf{G}(\boldsymbol{\Lambda})\}^{\mathrm{T}}\{\mathbf{F}-\mathbf{G}(\boldsymbol{\Lambda})\}+\left(\frac{1}{\alpha}-1\right) \frac{1}{k_{o}}\left\{\overline{\mathbf{F}}_{\text {int }}\right\}^{\mathrm{T}}\left\{\overline{\mathbf{F}}_{\text {int }}\right\}\right),
$$

where $\overline{\mathbf{F}}_{\text {int }}$ is the vector that collects the internal forces for each DOF except those supported by the nodes that are concerned with Dirichlet boundary conditions. $m_{o}$ and $k_{o}$ are appropriate normalization constants. They are set to the values of the optical and mechanical part of the functional, respectively, for the first guess of the displacement. $\alpha$ is a penalty parameter that allows to enforce the regularization of the DIC residual by the balance of momentum. From the DIC viewpoint, the mechanical residual introduces a length scale (depending on the penalty parameter $\alpha)$ in the minimization. This length scale is interpreted as a cut-off wave length of a filter applied to the measured displacement field. This filter being a 'mechanical' one, we see it as the most appropriate one. On the other hand, from the numerical simulation point of view, this mixed residual enables us to compute a displacement field that is driven in the bulk (without boundary conditions) by DIC, the optical residual acting as the volume 'optical driving force'.

Figure 8 shows the algorithm for the I-MIC procedure. An iterative procedure is initialized from an initial guess of the displacement and constitutive parameters. At each iteration, the 


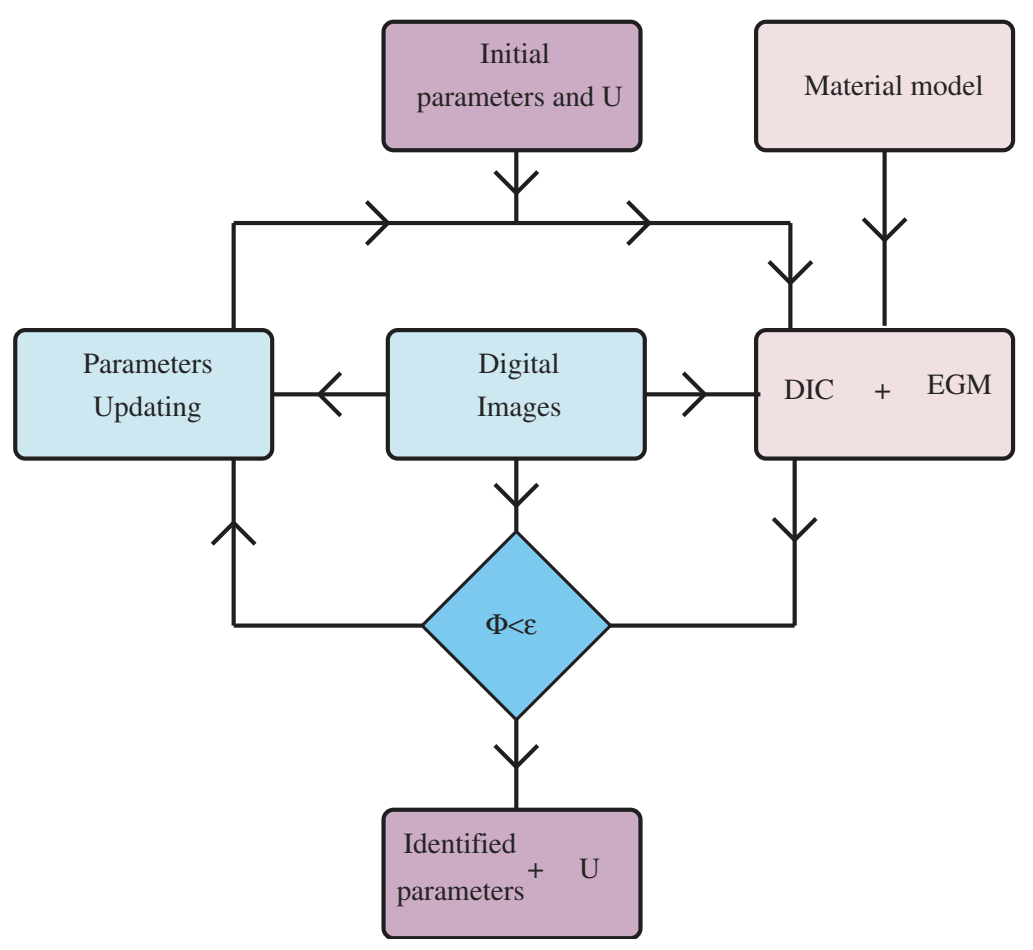

Figure 8. Integrated Mechanical Image Correlation algorithm. Note that the digital images are used at each step of the procedure (computation of the displacement field, evaluation of the cost function, and parameters updating) and that no boundary condition is required.

EGM stands for Equilibrium Gap Miminization.

displacement is evaluated for the current constitutive parameters by minimizing the mixed residual of Equation (30). For this purpose, a sequence of the following linear system is solved:

$$
\left[\mathbf{M}_{\mathrm{I}-\mathrm{DIC}}\right]\{\mathrm{d} \mathbf{U}\}=\left\{\mathbf{b}_{\mathrm{I}-\mathrm{DIC}}\right\},
$$

with (using the same modification as for DIC)

$$
\left[\mathbf{M}_{\mathrm{I}-\mathrm{DIC}}\right]=\frac{1}{m_{o}}[\mathbf{N}]^{\mathrm{T}}[\nabla \mathbf{F}][\nabla \mathbf{F}][\mathbf{N}]+\left(\frac{1}{\alpha}-1\right) \frac{1}{k_{o}}[\overline{\mathbf{K}}]^{\mathrm{T}}[\overline{\mathbf{K}}]
$$

where $[\overline{\mathbf{K}}]$ is the tangent stiffness matrix for the DOF that are concerned with the minimization of their associated internal forces. The right-hand side of Equation (31) is:

$$
\left\{\mathbf{b}_{\text {I-DIC }}\right\}=\frac{1}{m_{o}}[\mathbf{N}]^{\mathrm{T}}[\nabla \mathbf{F}]\{\mathbf{F}-\mathbf{G}\}-\left(\frac{1}{\alpha}-1\right) \frac{1}{k_{o}}[\overline{\mathbf{K}}]^{\mathrm{T}}\left\{\overline{\mathbf{F}}_{\mathrm{int}}\right\} .
$$

In practice, even for values of $\alpha$ close to 1 a strong reduction of the internal forces is obtained, meaning that the emphasis is on the mechanical part of the cost function. As a consequence, the norm of $\overline{\mathbf{F}}_{\text {int }}$ vanishes even for non-optimal constitutive parameters. This means that the variation of $\boldsymbol{\Lambda}$ can only influence the optical part of the cost function. The solution increment for the constitutive parameters is obtained by minimizing the DIC residual only. For this purpose, we use 
exactly the MIC equations (25), (27), and (28):

$$
\left[\mathbf{M}_{\mathrm{MIC}}\right]\{\mathrm{d} \boldsymbol{\Lambda}\}=\left\{\mathbf{b}_{\mathrm{MIC}}\right\} .
$$

Note that the term $[\Delta \mathbf{V} / \Delta \mathbf{\Lambda}]$ that is involved in the expression of $\mathbf{M}_{\text {MIC }}$ and $\mathbf{b}_{\text {MIC }}$ is substituted by $[\Delta \mathbf{U} / \Delta \boldsymbol{\Lambda}]$. In the present single step strategy, the sensitivity matrix $\Delta \mathbf{U} / \Delta \Lambda$ is computed with $\mathbf{U}$ fields that are obtained by solving Equation (31). Thus, no boundary conditions are invoked in the I-MIC approach which will be shown to be of great importance in the following.

The sensitivity analysis for the constitutive parameters leads to the following expression of the uncertainty:

$$
\left\langle\{\boldsymbol{\delta} \boldsymbol{\Lambda}\}\{\boldsymbol{\delta} \boldsymbol{\Lambda}\}^{\mathrm{T}}\right\rangle=2\left[\mathbf{M}_{\mathrm{MIC}}\right]^{-1} \sigma^{2} .
$$

Here again, the noise sensitivity is formally optimal but in practice it may differ from FEMU-R and MIC due to the solution history. It will be shown in the examples that not only is an optimal noise sensitivity obtained but also mesh independence.

\subsection{Identification of a steel plate's Poisson's ratio}

The example of the identification of the Poisson's ratio of a steel plate is proposed herein using the I-MIC strategy.

Figure 9 shows the deformed mesh and the longitudinal strain field. The I-MIC displacement is quite noisy on the edges where the minimization of the internal forces was not prescribed. However, this noise on the boundaries does not affect the identification of $v$. Figure 10 shows the evolution of the identified Poisson's ratio as a function of the mesh size obtained with I-MIC (with $\alpha=10^{-6}$ ). Error bars correspond to an image noise $\sigma$ of 2 gray levels for 8-bit digitization. A good agreement is obtained with the results of the previous section. The values of the identified Poisson's ratios are stable with a value of $0.285 \pm 0.014$ for an image noise $\sigma$ of 2 gray levels and 10-pixel elements. For this element size, a correlation error of $0.88 \%$ of the image dynamic range is obtained as for MIC. The evolution of the sensitivity to noise presented in Figure 10(b) shows that I-MIC leads to results similar to MIC i.e. with an optimal noise sensitivity as it was expected. The noise sensitivity has also very small variation when the mesh size is decreased. This means that the proposed I-MIC strategy is robust with respect to image noise and also to displacement noise.

Figure 11 shows the influence of the penalty parameter $\alpha$ on the results obtained with I-MIC. It is observed that when decreasing the element size a higher regularization (lower $\alpha$ ) is required to get stable results for this example where very low strain levels are involved. However the variation of $v$ with respect to the weighting parameter are low (less than 0.03).

Finally, I-MIC allows for the identification of constitutive parameters with an optimal noise sensitivity. As no boundary conditions are required, mesh independence is obtained. Concerning computational time, for FEMU, FEMU-R, and MIC, the computing time for DIC must be added to the time spent for identification, which gives a total time similar to the computational time for I-MIC.

\subsection{Identification of the damage law of a composite material}

The second example is dedicated to the identification of the damage law of a composite material. This example has already been studied in the literature in Claire et al. [19] and Roux and Hild [3] 


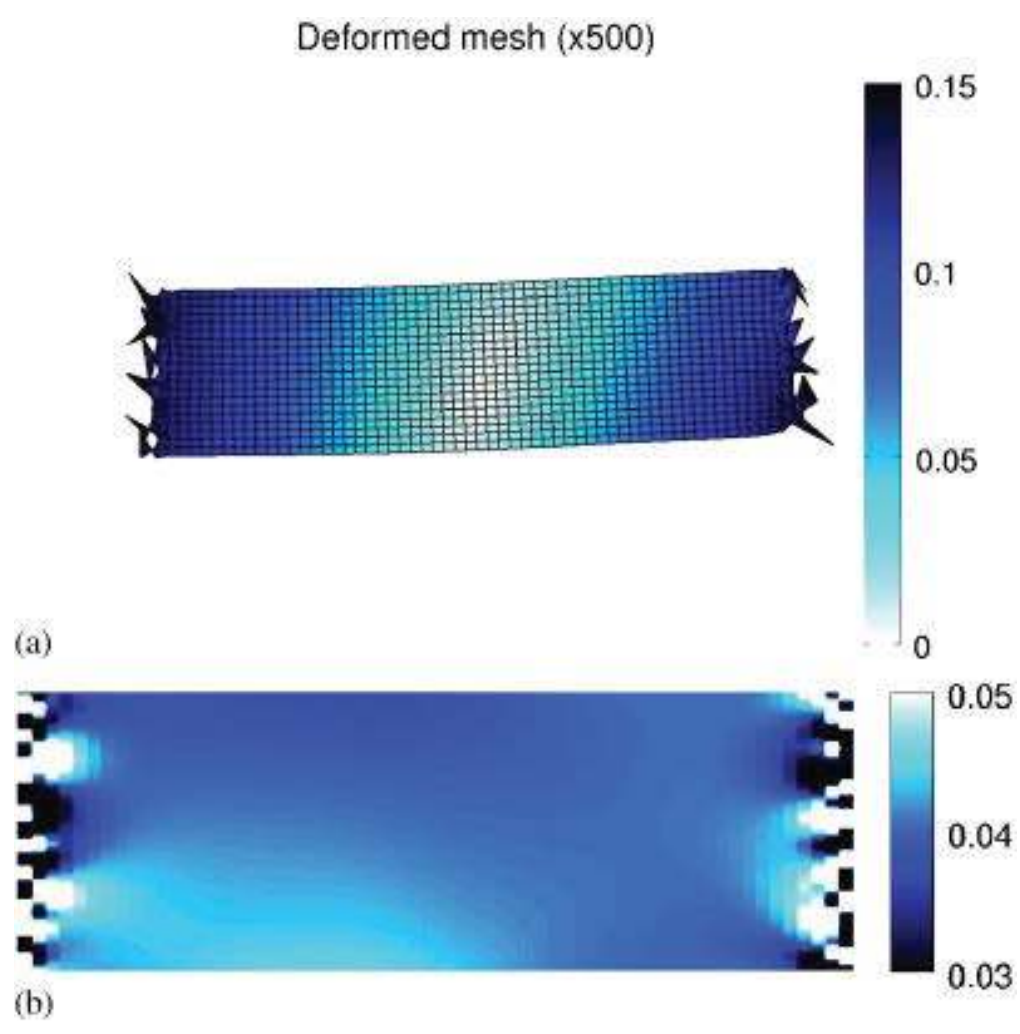

Figure 9. Displacement norm in pixel on the deformed mesh $(\times 500$ amplification $)$ and longitudinal strain field (in per cent) using 10-pixel elements and I-MIC analysis.

using the equilibrium gap method. Note that in Avril et al. [1], the elastic properties obtained using different identification strategies are compared.

The cruciform specimen (see Figure 12(a)) is made of a vinylester matrix reinforced by E-glass fibers. The physical pixel size is $68 \mu \mathrm{m}$ and the specimen thickness $3.53 \mathrm{~mm}$. A quasi-uniform distribution of orientations leads to an isotropic elastic behavior prior to matrix cracking and fiber breakage, which are the main damage mechanisms. The cross-shaped specimen is loaded in a multiaxial testing machine. The experiment is performed in such a way that the forces applied along two perpendicular directions are supposed to be identical. Their average is denoted by $F$. Eleven different load levels are analyzed, namely, $F=1-11 \mathrm{kN}$ with respect to the reference for no applied load. The failure load corresponded to $F=11.1 \mathrm{kN}$ (Figure 12(c)) just after the image at $F=11 \mathrm{kN}$ was recorded. Images have 8-bit digitization. Poisson's ratio obtained with I-MIC $(v=0.31$ ), using the first load state $F=1 \mathrm{kN}$, is in good agreement with the results found in the literature. For this load level, Young modulus is also identified. A value of $10 \mathrm{GPa}$ is obtained by adjusting the mean of the $x$ and $y$ components $\left(F_{x}\right.$ and $\left.F_{y}\right)$ of the resulting force computed by the I-MIC analysis to $1 \mathrm{kN}$. Again, this result is in good agreement with the results in the literature. The analysis is not detailed herein as the previous example was already dedicated to the identification of elastic properties.

A classical thermodynamic framework is used to describe the degradation of the material. A state potential $\Psi$ of the following form is adopted:

$$
\Psi=(1-D) E: S: E,
$$




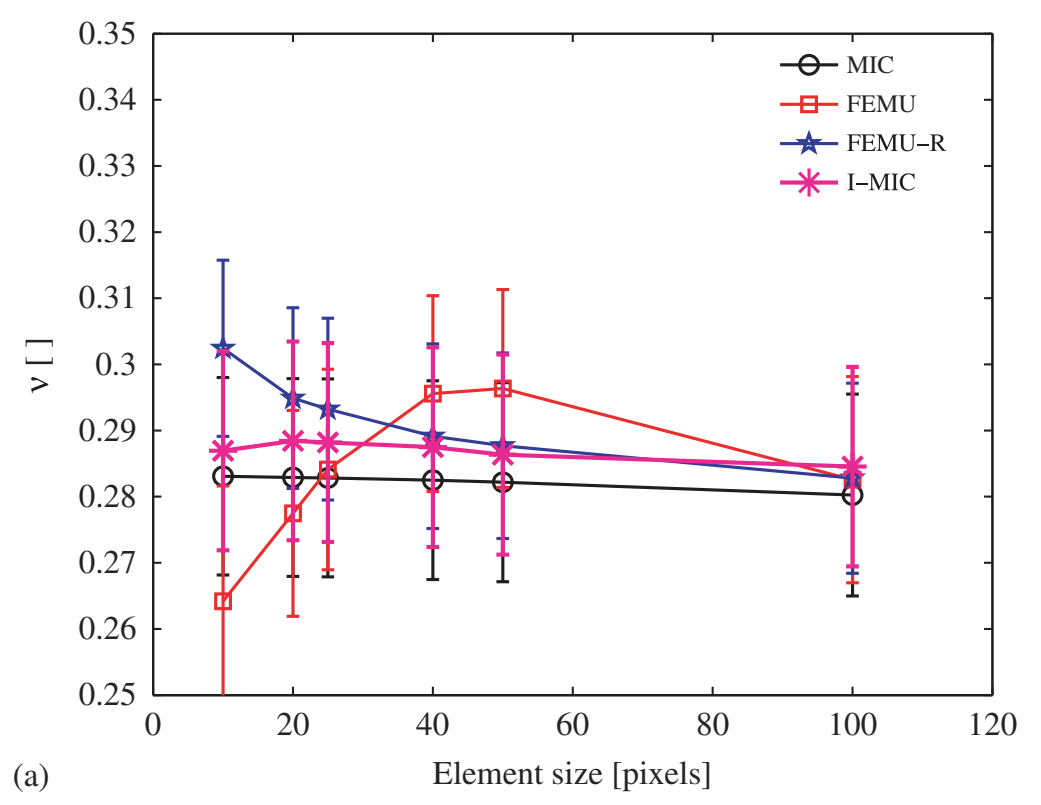

(a)

Element size [pixels]

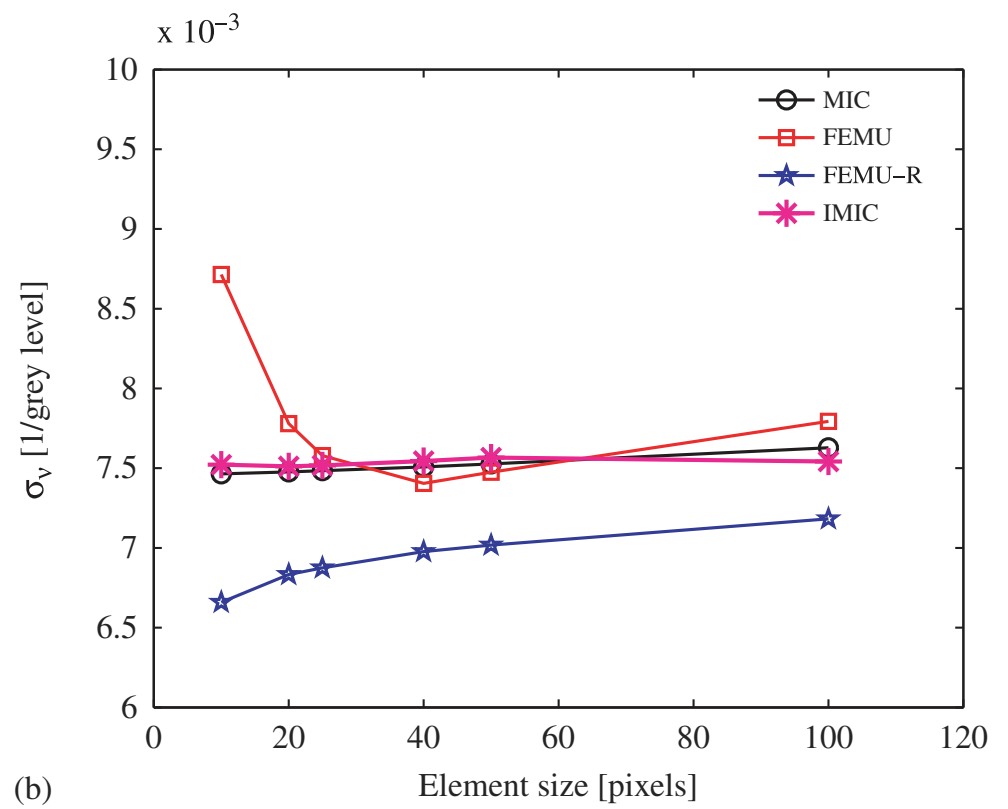

Figure 10. Evolution of the identified Poisson's ratio and its noise sensitivity as a function of the element size for the I-MIC strategy. The results are compared in the previous section with FEMU, FEMU-R, and MIC using Dirichlet boundary conditions from the DIC analysis with 200-pixel elements. Error bars correspond to an image noise of 2 gray levels.

where $E$ is the infinitesimal strain tensor, $S$ the Cauchy stress tensor, and $D$ the damage variable. The Clausius-Duhem inequality prescribes that the rate of damage must be non-negative. For this purpose, $D$ is written as a function of the maximum value of a positive equivalent strain $E_{\text {eq. }}$. In the present example, the equivalent strain, in plane stress, is chosen of Marigo's type [20]:

$$
E_{\mathrm{eq}}=\left[\left\langle E_{1}\right\rangle_{+}^{2}+\left\langle E_{2}\right\rangle_{+}^{2}+2 v\left\langle E_{1}\right\rangle_{+}\left\langle E_{2}\right\rangle_{+}\right]^{1 / 2}
$$




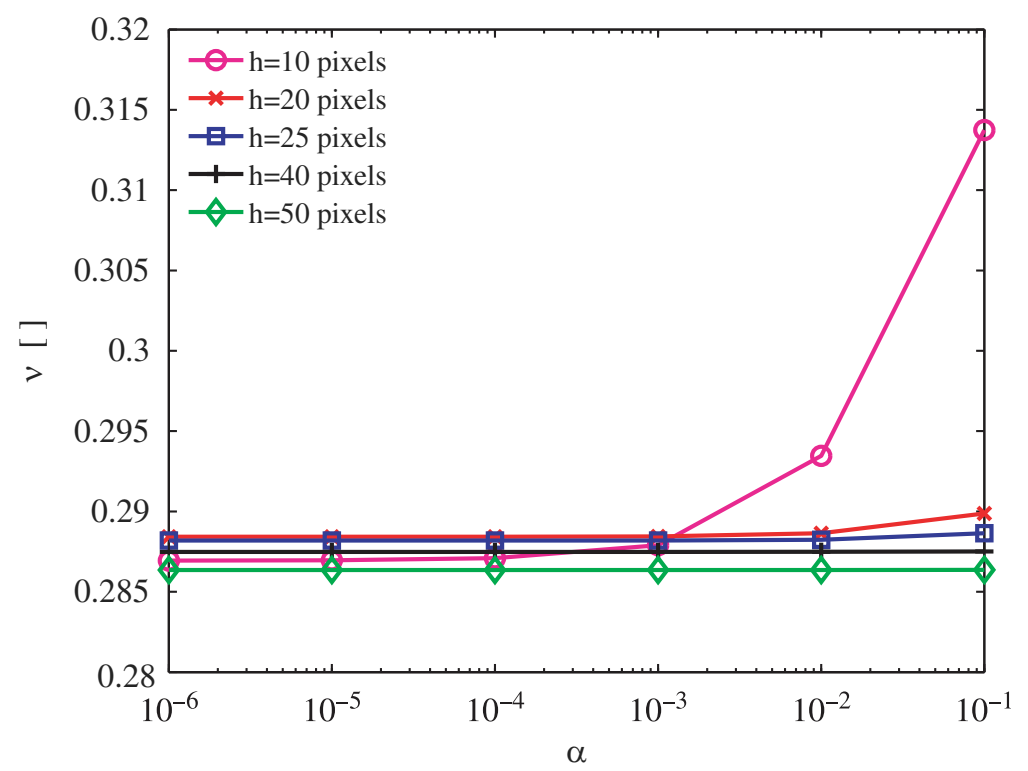

Figure 11. Evolution of the identified Poisson's ratio as a function of the penalty parameter $\alpha$ for different values of the element size.

where $E_{1,2}$ are the principal strains and $\langle\bullet\rangle_{+}$the Macauley brackets (i.e. the positive part of the inner argument). For identification purposes, the damage law will be searched for an exponential form:

$$
D=1-\mathrm{e}^{-\hat{E}_{\mathrm{eq}} / E_{\mathrm{c}}}
$$

where $E_{\mathrm{c}}$ is a constant to be identified and $\hat{E}_{\text {eq }}$ denotes the maximum value reached by $E_{\text {eq }}$ until the current time.

The design of the cross-shaped specimen requires that the circular contours of the sample are properly described by the mesh around the strain localization area. This is difficult to obtain with structured meshes even if a masking of void area is adopted. Further, to properly capture the strain localization, one would refine the mesh around the corners of the specimen. This is more easily achieved with an unstructured mesh of triangular elements. For this purpose, the unstructured meshes shown in Figure 13 are used in the following. The mean element sizes for these meshes are 20,15, and 10 pixels. They consist in 4500, 7500, and 17000 piecewise linear elements, respectively. Appendix describes the numerical integration strategy that was used for unstructured meshes. Figures 14(a),(b) show, respectively, the horizontal displacement field and the von-Mises strain field obtained by a pure DIC analysis using a mean element size of 15 pixels at the last load level $F=11 \mathrm{kN}$. Owing to geometrical and/or material imperfection, strain and damage localization are observed in the bottom left corner of the specimen. However, high strain levels are also observed in the other three corners.

Because of this loss of symmetry, any approaches using boundary conditions will fail. Indeed, this information (loss of symmetry and strain localization in the bottom left corner) is not contained in the boundary conditions (even if they are extracted from DIC) that should be applied on the four edges of the samples as shown in Figure 15. This figure allows to compare the result of a I-MIC analysis with a fixed damage parameter $\left(E_{\mathrm{c}}=0.04\right)$ and the result of a numerical simulation with DIC boundary conditions and the same value for the damage parameter. We observe that 




(a)

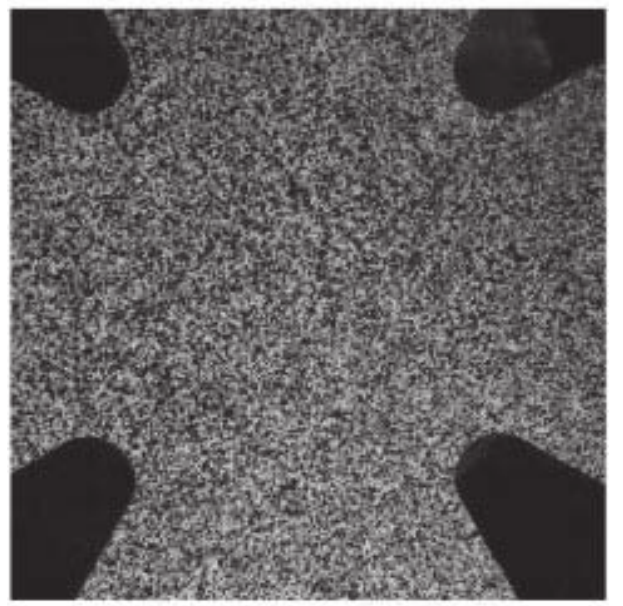

(b)

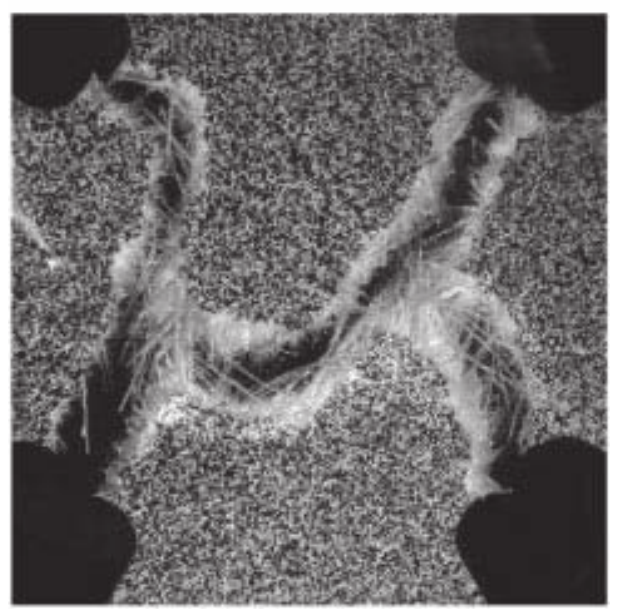

(c)

Figure 12. Initial image of the cruciform composite specimen (a). (b) and (c) show the specimen at a load of $11 \mathrm{kN}$ and after failure. 


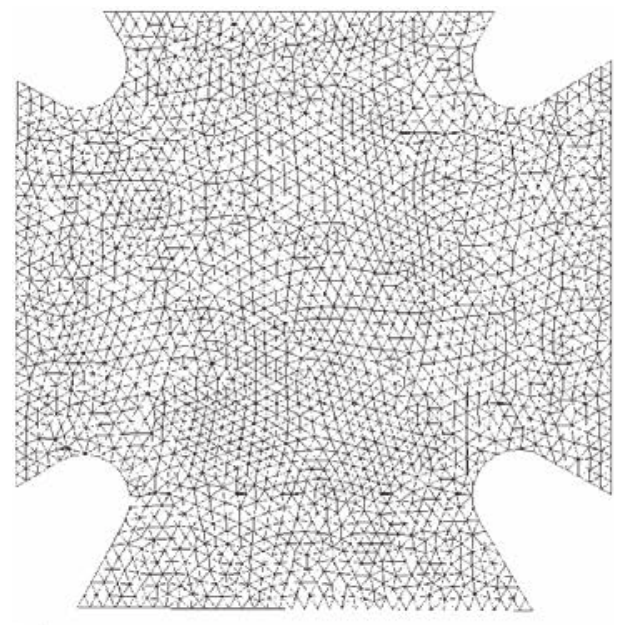

(a)

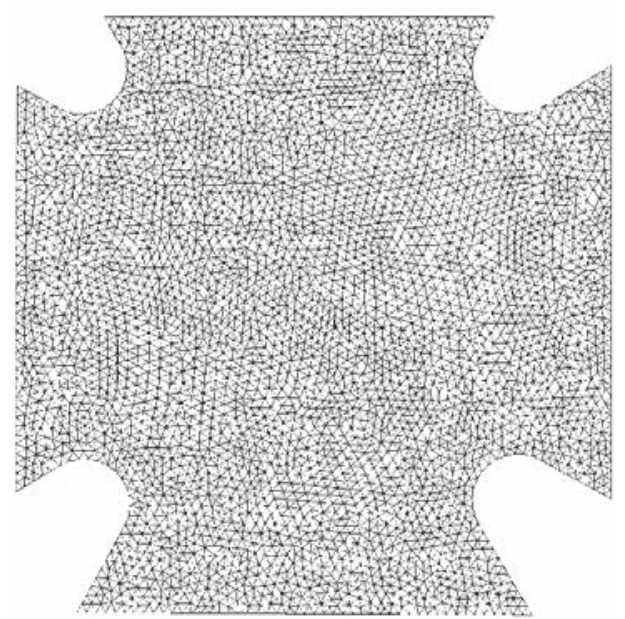

(b)

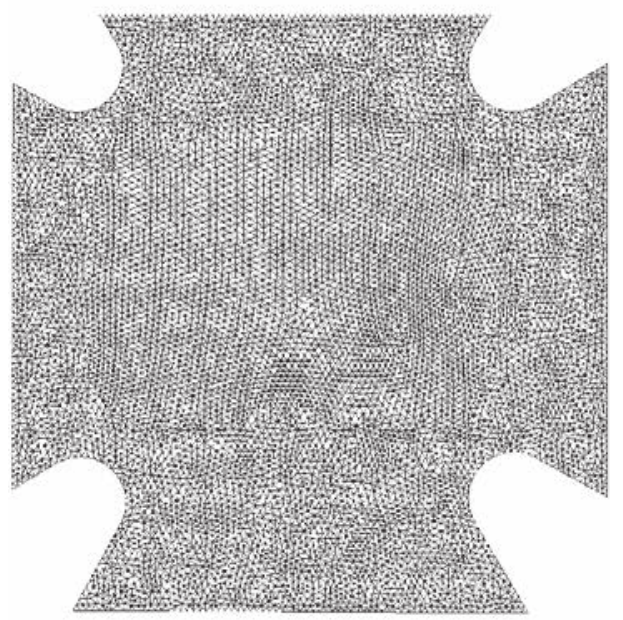

(c)

Figure 13. Various meshes used for the I-MIC analyses. 


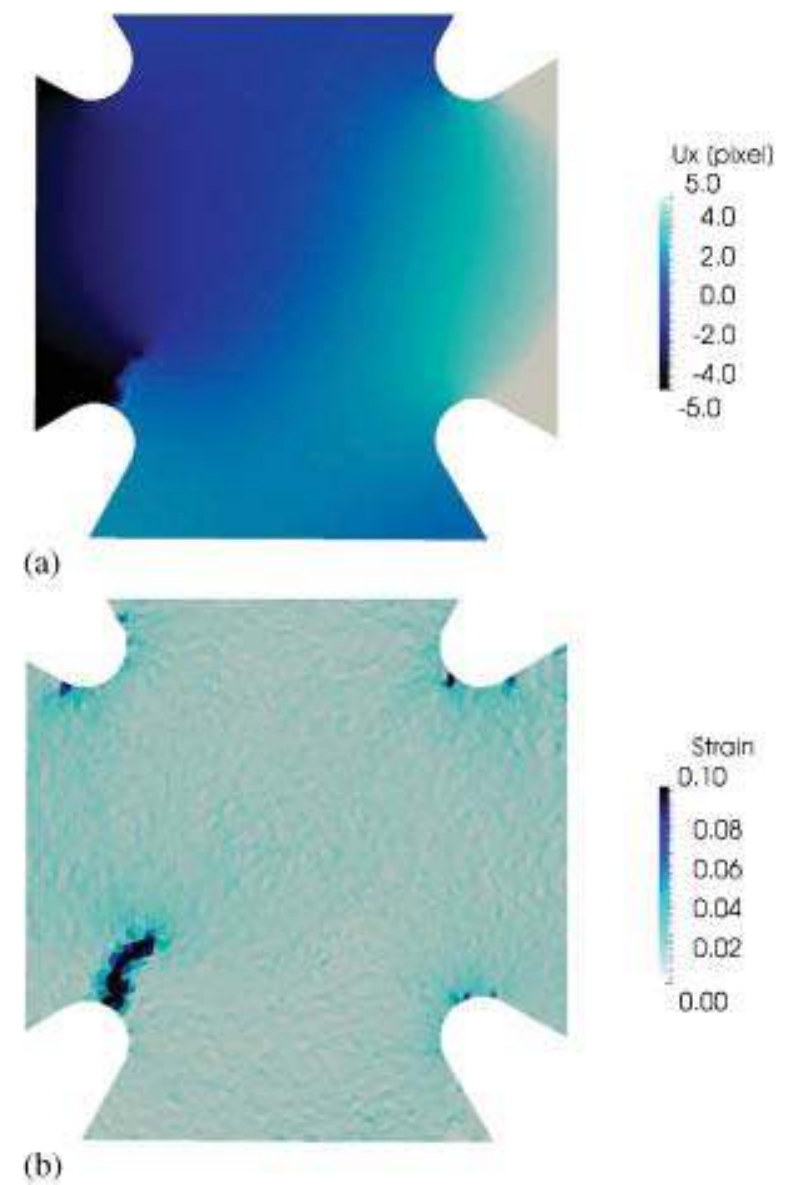

Figure 14. Horizontal displacement (in pixel) and von-Mises strain obtained with a DIC analysis at the last load level $F=11 \mathrm{kN}$.

strain localization is obtained with I-MIC at the bottom left corner of the specimen, whereas the numerical simulation with experimental boundary conditions leads to strain concentrations only. Therefore, identification procedures using boundary conditions are not appropriate in the present case. The proposed I-MIC strategy is the only FEMU-based strategy that does not require boundary conditions and is thus appropriate. For this reason, the I-MIC strategy only is investigated in this example. The mixed residual in I-MIC acts as 'volume' conditions coming from the mismatching of the images that are the data that contain the information about the strains localization. Note that direct identification techniques, such as the equilibrium gap method in Claire et al. [19] and Roux and Hild [3] or the Virtual Field Method in Chalal et al. [21, 22], can also be used.

For the identification of the damage law all the images for F ranging between 1 and $11 \mathrm{kN}$ are used. For this purpose, a time integral is added in the I-MIC functional. In the present example the material parameter that is searched for is $E_{\mathrm{c}}$. The I-MIC analyses are carried out for varying penalty parameter and varying mesh size. Figure 16(a) shows the evolution of the identified damage parameter as a function of the penalty parameter for different mesh sizes. As for the case of the identification of Poisson's ratio, the identified $E_{\mathrm{c}}$ has no mesh dependence. There is no discrepancy of the identification for all the mesh sizes and all the penalty parameter values. We observe only a low decrease when the penalty parameter is decreasing, irrespective of the element size. A converged value of 0.0262 is obtained. The low sensitivity to noise is illustrated in Figure 16(b). 


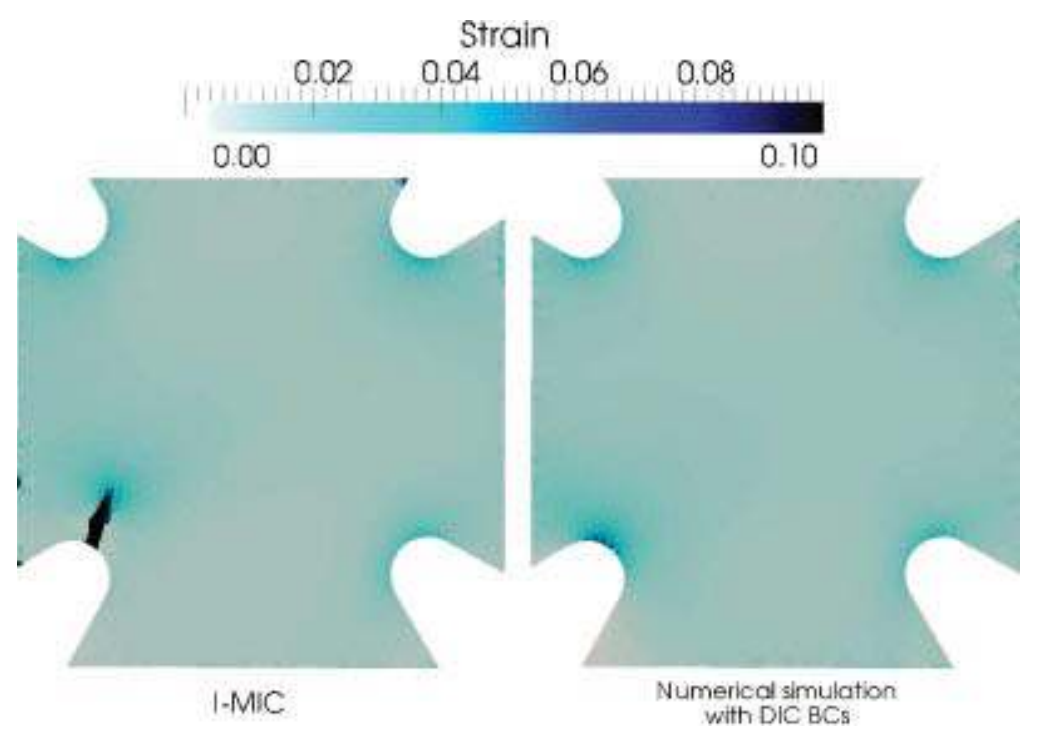

Figure 15. Von-Mises strain field with a mesh size of 15 pixels for the last load level $F=11 \mathrm{kN}$ : result obtained by I-MIC with fixed constitutive parameter $\left(E_{\mathrm{c}}=0.04\right)$ and penalty parameter $\alpha$ set to $10^{-3}$ is compared with the result of a numerical simulation with DIC boundary conditions and the same constitutive parameter $\left(E_{\mathrm{c}}=0.04\right)$.

The same trend as in Figure 16(a) is observed. The sensitivity is about $1.5 \times 10^{-6}$ per gray level which is about $0.5 \times 10^{-4}$ the identified value.

Figure 17 shows the displacement field (a) and von-Mises strain field (b) for the I-MIC analysis using a mesh with a mean element size of 15 pixels and a penalty parameter $\alpha$ set to $10^{-3}$. We observe strong similarities between Figure 17 and Figure 14. This shows how the whole kinematic of the test is captured by this fully coupled analysis (I-MIC). In addition, for this value of the penalty parameter $\alpha$, the mean optical residual is $0.69 \%$ of the image dynamic for DIC and $0.8 \%$ for I-MIC. Therefore, the mechanical regularization does not deteriorate the minimization of the optical part of the I-MIC functional. However, the norm of the internal force vector on the interior nodes $\overline{\mathbf{F}}_{\text {int }}$ is reduced by a factor of $10^{4}$ compared to the internal forces computed directly from the DIC displacement. This confirms that the I-MIC displacement field has been mechanically filtered.

Figure 18 shows the von-Mises strain field and the damage field at the last load level for 15-pixel elements and $\alpha$ set to $10^{-1}, 10^{-3}$, and $10^{-6}$. The highest $\alpha$ gives results similar to a DIC analysis (see Figure 14). Decreasing $\alpha$ increases the mechanical filtering and thus allows for a higher resolution. Failure initiation is predicted also at the top corners and at the bottom right corner exactly as observed in the image of the sample after failure (Figure 12(c)). These differences are observed between $\alpha=10^{-1}$ and $\alpha=10^{-3}$, the results for $\alpha=10^{-3}$ and $\alpha=10^{-6}$ are similar. This can be related to the variation of the identified damage parameter with $\alpha$ obtained in Figure 16(a). Decreasing $\alpha$ leads to lower values of $E_{\mathrm{c}}$ and earlier failure initiation. Note that once the proper failure mode has been captured by the I-MIC analysis, very low differences in the identified damage parameter and in the strain field are observed when varying $\alpha$.

Figure 19 shows the von-Mises strain field and the damage field at the last load level for the three meshes presented in Figure 13 and a penalty parameter $\alpha=10^{-3}$. As expected, no mesh dependence is obtained. The strain (and damage) localization is more pronounced at the bottom 



Figure 16. Comparison of the identified damage parameter (a) and its sensitivity to noise (b) as a function of the penalty parameter $\alpha$ for different mesh sizes.

left corner than at the other three corners. The strain localization is obtained at the same position, within a zone of a similar shape and size. This confirms that no mesh bias is obtained using the proposed I-MIC strategy.

In Figure 20(a), we compare the mean resulting force $\left(F_{x}+F_{y}\right) / 2$ from the load cells and from the I-MIC analyses for 15-pixel elements and varying $\alpha$. These resulting forces are computed from the stress field integrated along lines parallel to the edges of the specimen. $F_{x}$, respectively $F_{y}$, is given by the mean of the amplitude of the resulting force along the $x$, respectively $y$, direction between the left, respectively top, and the right, respectively bottom, edges. The global response is well described by I-MIC. Again, we obtain very low variations with respect to $\alpha$. This figure 


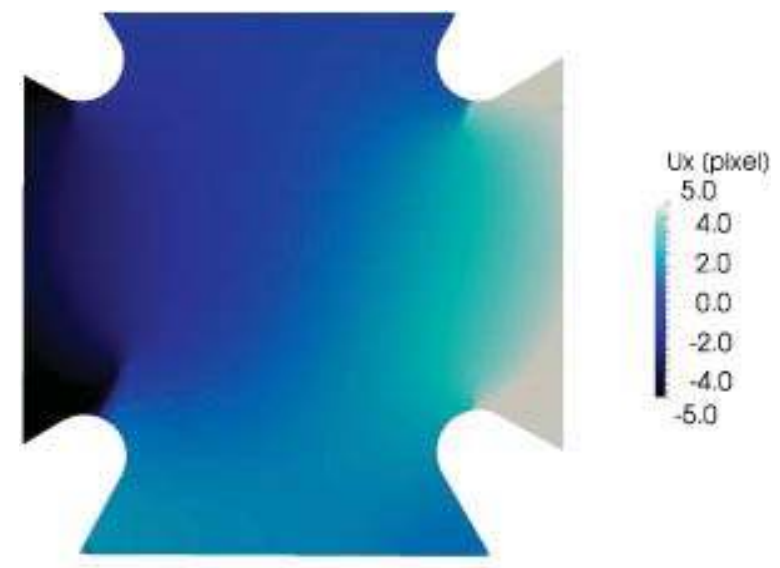

(a)

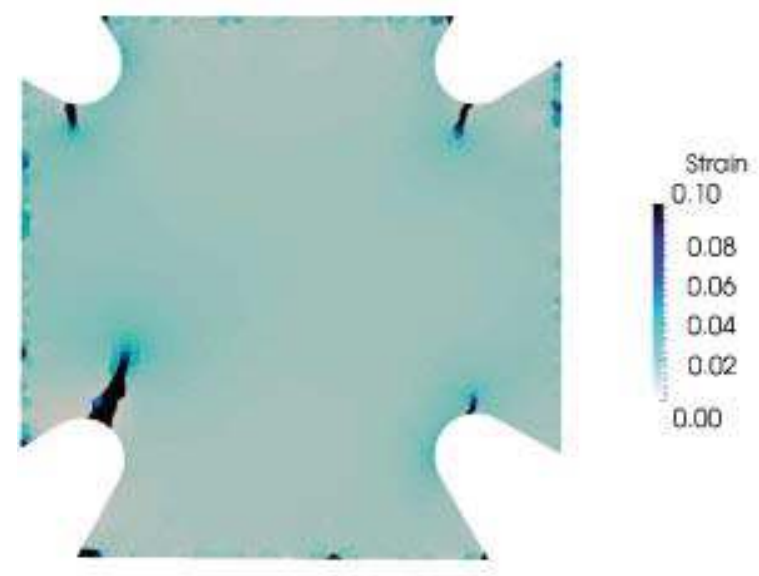

(b)

Figure 17. Horizontal displacement (in pixel) and von-Mises strain obtained with an I-MIC analysis with $\alpha=10^{-3}$ at the last load level.

shows that with the proposed identification strategy that extracts constitutive parameters directly from digital images, the global behavior of the sample is also captured. Figure 20(b) shows the $x$ and $y$ components of the resulting force for I-MIC with 15-pixel elements and $\alpha=10^{-3}$. We observe that $F_{x}$ is slightly higher than $F_{y}$. A difference of about $3 \mathrm{kN}$ is obtained for the last load level. This difference can be related to the failure patterns presented above. Indeed, strain localization and failure initiation is obtained (in Figure 19 for example) along paths orthogonal to the $x$-axis. Using the global data available (the mean resulting force $F$ ), failure patterns at $45^{\circ}$ would have been predicted whereas more less vertical cracks are observed in the image of the sample after failure (Figure 12(c)). Extracting the displacement field and the constitutive parameters directly from the digital images appears again as an essential feature of the proposed I-MIC strategy.

As for the previous example, the I-MIC strategy that is fully coupled and does not require boundary conditions allows us to capture phenomena that cannot be represented using a standard FE analysis even with DIC boundary conditions. The 'optical driving forces' arising from the optical part of the functional drive the mechanical part to the actual kinematics. We do not observe mesh dependence either on the displacement fields or on the strain field or on the damage field or on the resulting forces. The variations with respect to the penalty parameter are also really low. 


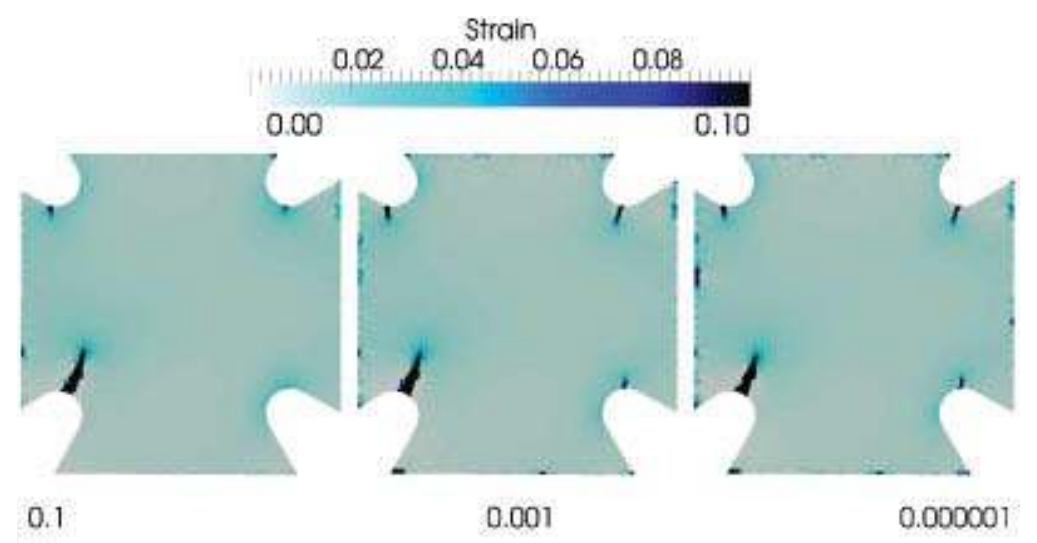

(a)

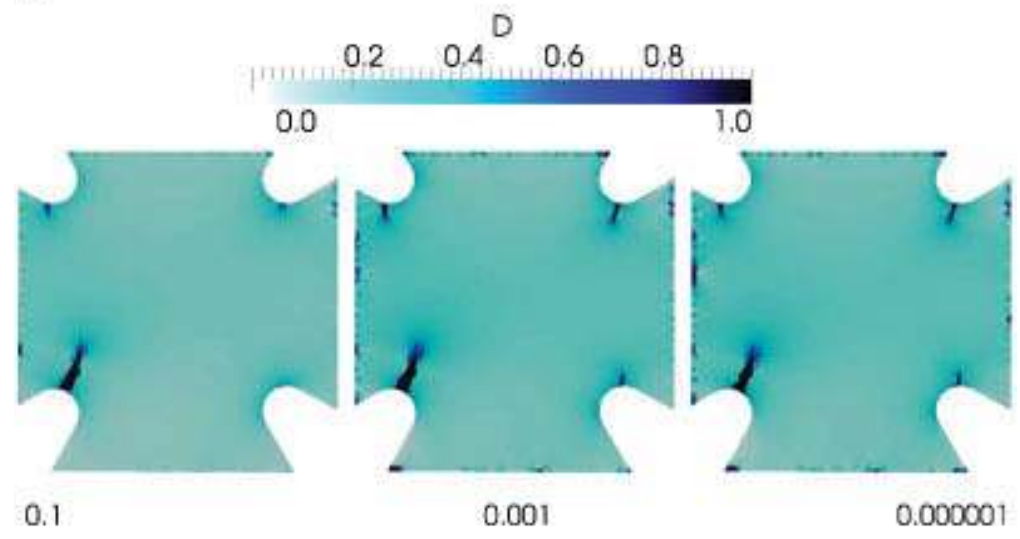

(b)

Figure 18. Comparison of the von-Mises strain field (a) and damage field (b) at the last load level for I-MIC analyses with different values of $\alpha$.

As these parameters (element size and penalty parameter) were the only two tuning parameters of the proposed approach, and as no discrepancy is obtained when these parameters are varying, we conclude that I-MIC leads to an objective identification of constitutive parameters.

Finally, the damage model used herein does not use any regularization, such as gradient models [23], non-local models [24], or delay effect [25]. However, the I-MIC analysis does not exhibit any spurious numerical strain localization that should have been obtained in an FE analysis without regularization of the damage model. Again, the length scale in the I-MIC functional is the key feature. This length scale was seen above as a cut-off wavelength of a mechanical filter that assists DIC analysis or that allows DIC to drive mechanics. Here, the length scale acts as a localization limiter in the same spirit as for the above-mentioned regularization techniques.

\section{CONCLUSION}

In this paper, we investigate noise sensitivity reduction techniques for constitutive parameters identification based on FEMU and a fully integrated approach. First we analyze the regularization proposed by Leclerc et al. [18]. Then we suggest a formulation based on the correlation residual (MIC) that allows to extract constitutive parameters directly from digital images. The strategy 


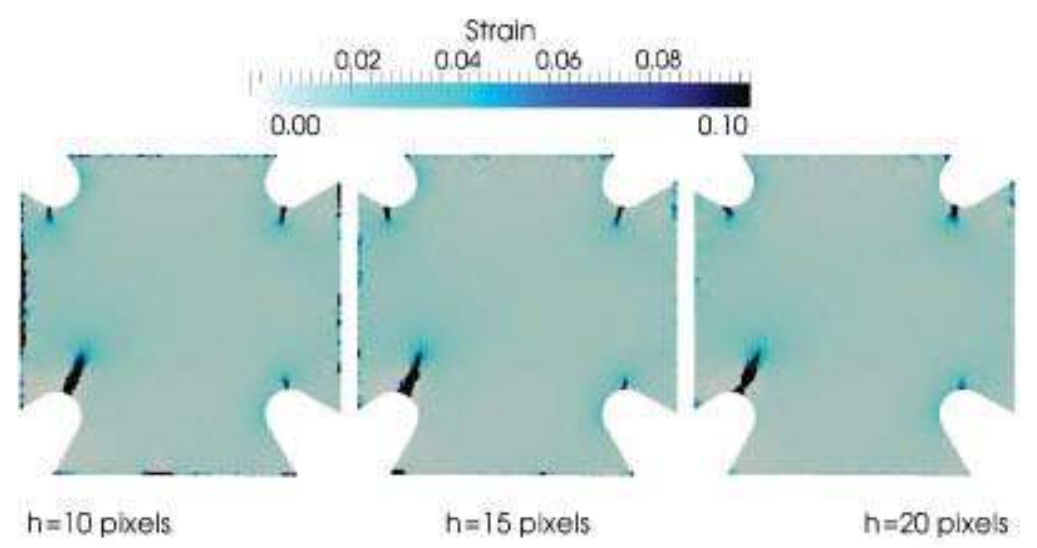

(a)

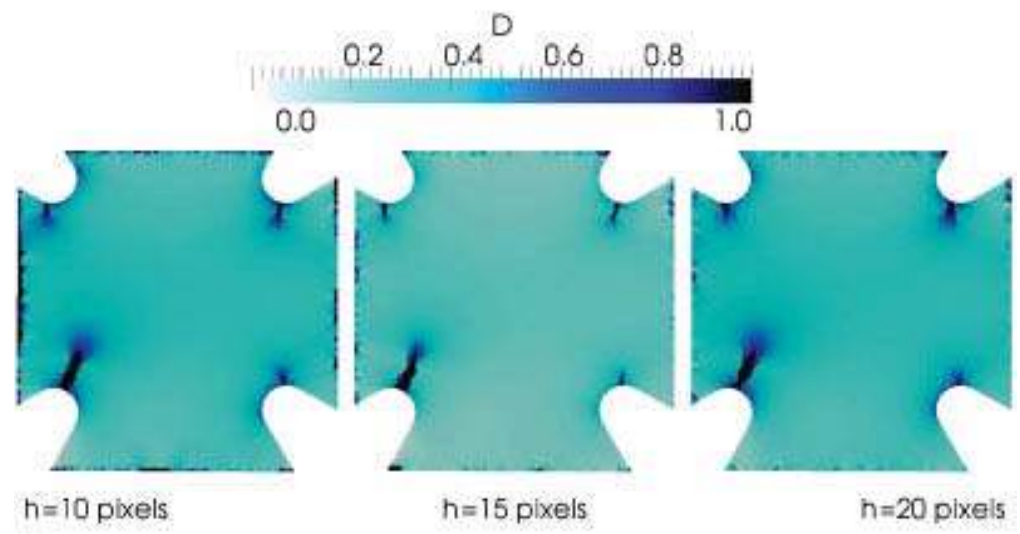

(b)

Figure 19. Comparison of the von-Mises strain field (a) and damage field (b) at the last load level for different mesh sizes for I-MIC analyses with $\alpha=10^{-3}$.

is optimal in terms of noise sensitivity. The noise sensitivity of the presented approaches are theoretically studied. A first application confirms the theoretical results about noise sensitivity and illustrates the difference between all the investigated techniques. We obtain that all these techniques exhibit strong dependence upon the boundary conditions that are used for the numerical simulation step.

To circumvent this dependence, a fully integrated strategy based on the minimization of a mixed optical/mechanical cost function is proposed (I-MIC). The latter is optimal in terms of noise sensitivity and does not require boundary conditions. This feature is shown to be of major interest. Indeed, for the first example, the I-MIC strategy is shown to have no mesh dependence as the displacement field is driven in the bulk by 'optical driving forces'. A second example is dedicated to the damage law identification of a composite material. The I-MIC identification leads to the determination of the parameters of a damage model with a low noise sensitivity. The full coupling between measurement and numerical simulations allows to capture the loss of symmetry of the test and the strain localization at the proper position without mesh bias.

In the proposed technique, the measurement is bidimensional and the mechanical fields are supposed to be constant through the thickness of the specimen. If one would like to use I-MIC to identify non-linear constitutive laws at large strains, the limit of the technique (those of other 2D methods also) in its present form may be reached. However, X-ray tomography is now commonly 

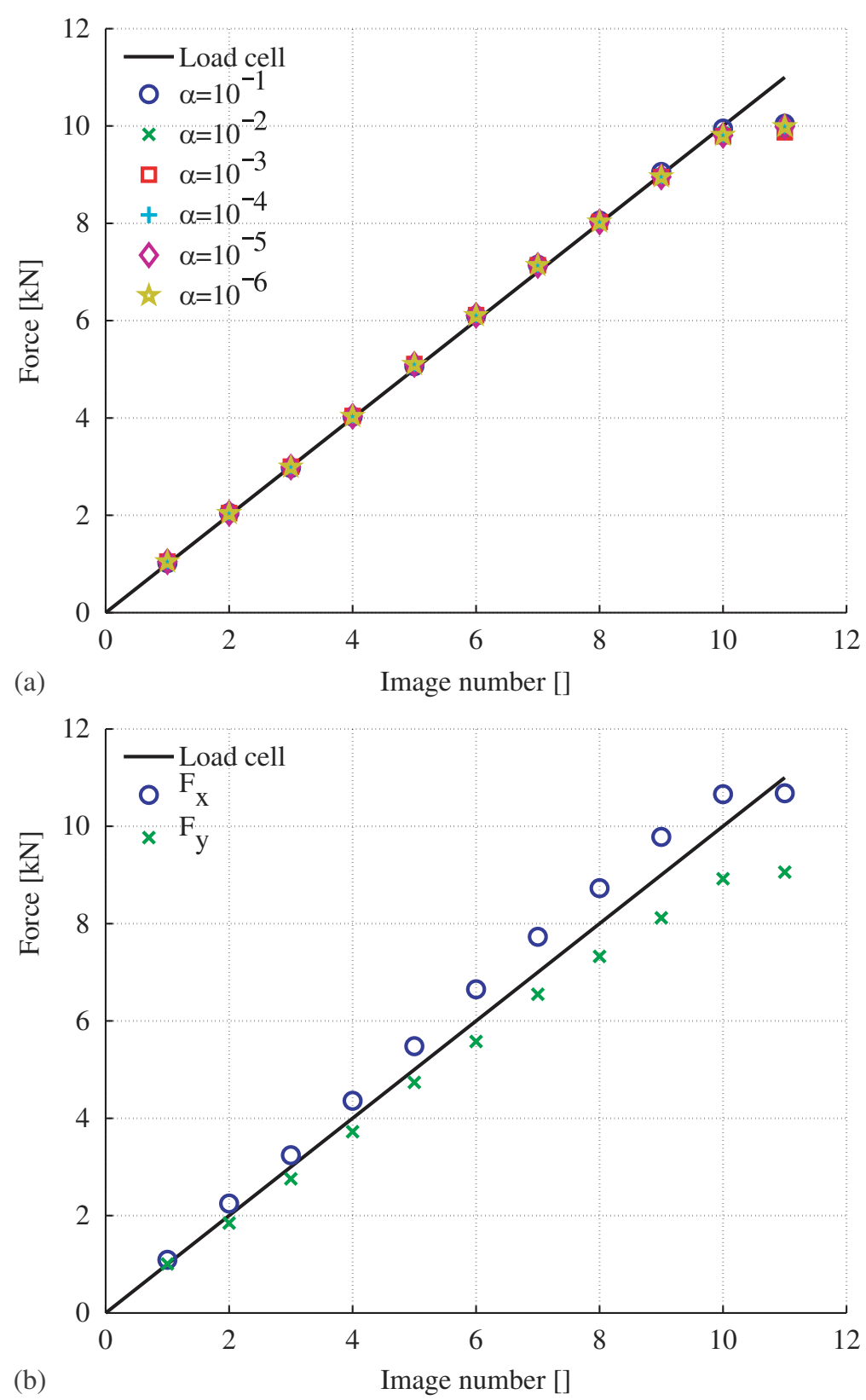

Figure 20. Comparison of the load cell data with the mean resulting force for different values of $\alpha$ and 15 pixel elements (a). Comparison of the load cell data with the $x$ and $y$ components of the resulting force for 15 pixel elements and $\alpha=10^{-3}$ (b).

available for research labs. Digital Volume Correlation has also been developed up to a point close to the achievement of DIC, see Rannou et al. [26]. I-MIC could thus be directly extended to fully $3 \mathrm{D}$ analysis for the identification for example of ductile failure models. If $3 \mathrm{D}$ imaging of the material is not possible, one could imagine to integrate the optical flow equation on the surface of a 3D model of the specimen, whereas the mechanical regularization is performed in the bulk. The extensions of the present work will be foreseen in the near future. 


\section{APPENDIX A}

In the last example of the paper, DIC analyzes are performed using an unstructured mesh of triangular elements (T3). The main advantage compared to structured mesh with quadrangular element (Q4) is that regions of interest of arbitrary geometry can be considered. However, a question arises concerning the integration of the DIC bilinear and linear forms. Using Q4 square element, the integration of $\left[\mathbf{M}_{\mathrm{DIC}}\right]$ and $\left[\mathbf{b}_{\mathrm{DIC}}\right]$ (or other similar terms) is usually performed pixelwise. In other words, the integrals are evaluated as discrete sums over the pixels of the ROI. Using T3 unstructured meshes, the same strategy can be used. In this case, the uncertainty is the same. Figure A1(a) shows the evolution of the measurement uncertainty for Q4 structured meshes and T3
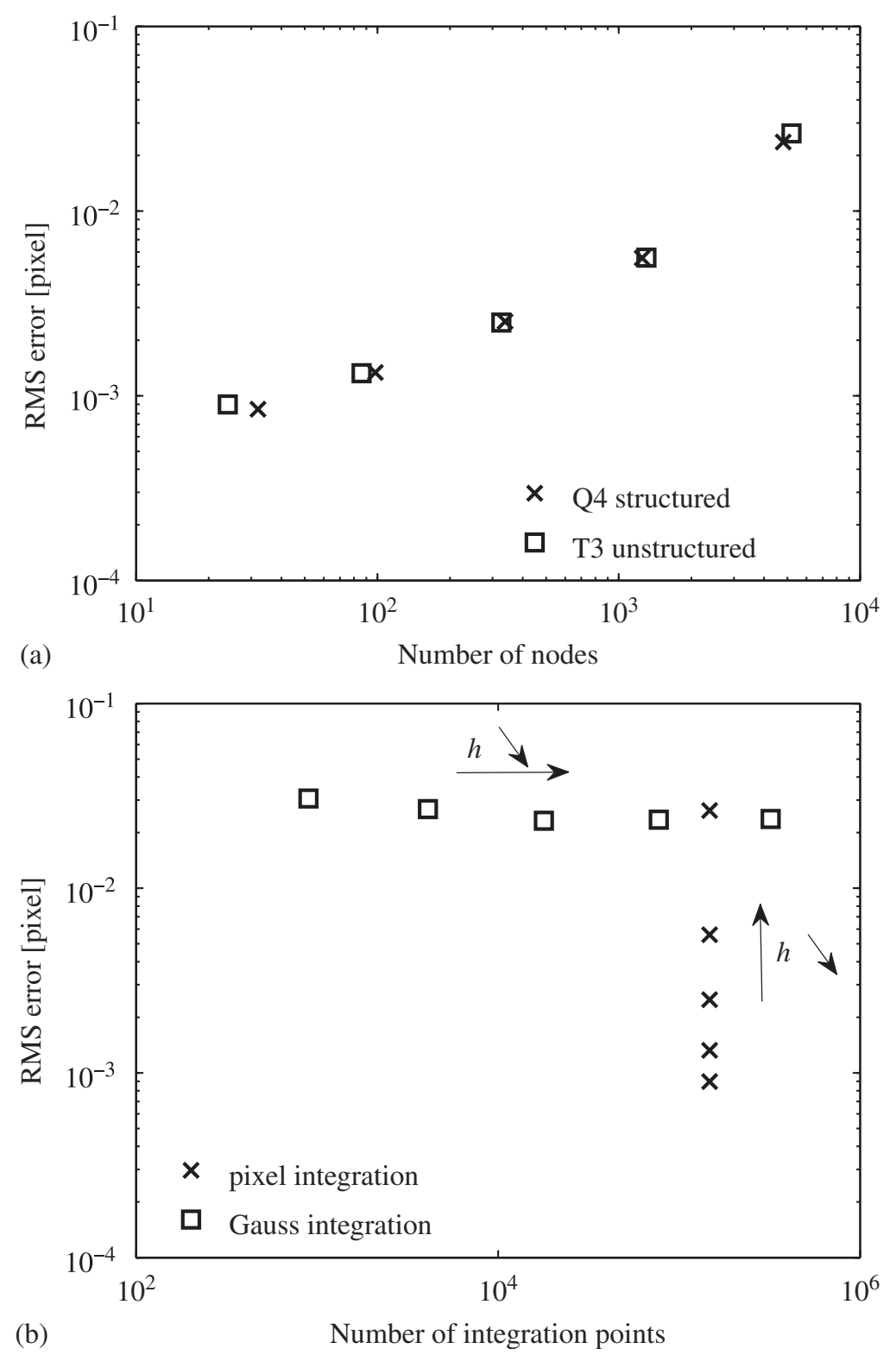

Figure A1. Evolution of the displacement uncertainty: (a) comparison of Q4 structured meshes and T3 unstructured mesh with pixel-wise integration and (b) comparison of pixel-wise integration and Gauss integration for $\mathrm{T} 3$ unstructured meshes. 
unstructured meshes. The uncertainty is computed as the standard deviation of the displacement field obtained by a DIC analysis between a reference image and the same image that was artificially translated by 0.5 pixel along $x$ and $y$. In this figure, the uncertainty is plotted as a function of the number of nodes in the mesh for varying element size. No difference is observed between the two calculations.

However, performing the inverse mapping from the co-ordinates of each pixel in the image frame to the reference frame of the T3 element may be computationally expensive. As an alternative, we suggest that for T3 unstructured meshes, the integration is performed using a Gauss rule with 64 points (derived from a rule for Q4 elements). Then, no inverse mapping is required. The drawback is that the gradient of the image (involved in $\left[\mathbf{M}_{\mathrm{DIC}}\right]$ and $\left[\mathbf{b}_{\mathrm{DIC}}\right]$ ) must be computed at integration points whose position is not an integer value. In the presented example, we use a spline cubic interpolation of the gray level from which the gradient is evaluated at the non-integer position. Figure A1(b) allows to compare the variation of the uncertainty as a function of the number of integration points for $\mathrm{T} 3$ unstructured meshes with the two integration rules. We conclude that when the element size is small enough such that the suggested Gauss integration rule provides approximately the same density of integration points, the results are identical.

\section{ACKNOWLEDGEMENTS}

Mecanium is gratefully acknowledged for having supplied the author for the images of the first example.

\section{REFERENCES}

1. Avril S, Bonnet M, Bretelle A, Grediac M, Hild F, Ienny P, Latourte F, Lemosse D, Pagano S, Pagnacco E, Allix O, Deü JF. Overview of identification methods of mechanical parameters based on full-field measurements. Experimental Mechanics 2008; 48(4):381-402.

2. Grédiac M, Pierron F, Avril S, Toussaint E. The virtual fields method for extracting constitutive parameters from full-field measurements: a review. Strain 2006; 42(4):233-253.

3. Roux S, Hild F. Digital Image Mechanical Identification (DIMI). Experimental Mechanics 2008; 48(4):495-508.

4. Avril S, Pierron F. General framework for the identification of constitutive parameters from full-field measurements in linear elasticity. International Journal of Solids and Structures 2007; 44(14-15):4978-5002.

5. Kavanagh K, Clough R. Finite element applications in the characterization of elastic solids. International Journal of Solids and Structures 1971; 7:11-23.

6. Lecompte D, Smits A, Sol H, Vantomme J, Van Hemelrijck D. Mixed numerical-experimental technique for orthotropic parameter identification using biaxial tensile tests on cruciform specimens. International Journal of Solids and Structures 2007; 44(5):1643-1656.

7. Genovese K, Lamberti L, Pappalettere C. Finite element analysis of a new customized composite post system for endodontically treated teeth. Journal of Biomechanics 2005; 38(12):2375-2389.

8. Levenberg K. A method for the solution of certain nonlinear problems in least squares. Quarterly of Applied Mathematics 1944; 2(2):164-168.

9. Oomens C, Ratingen M, Janssen J, Kok J, Hendriks M. A numerical-experimental method for a mechanical characterization of biological materials. Journal of Biomechanics 1993; 26:617-617.

10. Sutton M, Wolters W, Peters W, Ranson W, McNeill S. Determination of displacements using an improved digital correlation method. Image Vision Computing 1983; 1(3):133-139.

11. Besnard G, Hild F, Roux S. 'Finite-element' displacement fields analysis from digital images: application to Portevin-Le Châtelier bands. Experimental Mechanics 2006; 46(6):789-803.

12. Réthoré J, Hild F, Roux S. Extended digital image correlation with crack shape optimization. International Journal for Numerical Methods in Engineering 2007; 73(2):248-272.

13. Réthoré J, Elguedj T, Simon P, Coret M. On the use of NURBS functions for displacement derivatives measurement by digital image correlation. Experimental Mechanics 2009; DOI: 10.1007/s11340-009-9304-z. 
14. Réthoré J, Roux S, Hild F. Noise-robust stress intensity factor determination from kinematic field measurements. Engineering Fracture Mechanics 2006; 75(13):3763-3781.

15. Réthoré J, Hild F, Roux S. An extended and integrated digital image correlation technique applied to the analysis of fractured samples. European Journal of Computational Mechanics 2009; 18:285-306.

16. Réthoré J, Hild F, Roux S. Shear-band capturing using a multiscale extended digital image correlation technique. Computer Methods in Applied Mechanics and Engineering 2007; 196(49-52):5016-5030.

17. Réthoré J, Besnard G, Vivier G, Hild F, Roux S. Experimental investigation of localized phenomena using digital image correlation. Philosophical Magazine 2008; 88(28,29):3339-3355.

18. Leclerc H, Perie J, Roux S, Hild F. Integrated digital image correlation for the identification of mechanical properties. Computer Vision/Computer Graphics Collaboration Techniques. Springer: Berlin, 2009.

19. Claire D, Hild F, Roux S. A finite element formulation to identify damage fields: the equilibrium gap method. International Journal for Numerical Methods in Engineering 2004; 61:189-208.

20. Marigo J. Formulation dune loi dendommagement dun matériau élastique. Comptes Rendus de l'Accadémie des Sciences 1981; 292:1309-1312.

21. Chalal H, Meraghni F, Pierron F, Grédiac M. Direct identification of the damage behaviour of composite materials using the virtual fields method. Composites Part A 2004; 35(7-8):841-848.

22. Chalal H, Avril S, Pierron F, Meraghni F. Experimental identification of a nonlinear model for composites using the grid technique coupled to the virtual fields method. Composites Part A 2006; 37(2):315-325.

23. Peerlings R, De Borst R, Brekelmans W, De Vree J. Gradient enhanced damage for quasi-brittle materials. International Journal for Numerical Methods in Engineering 1996; 39(19):3391-3403.

24. Pijaudier-Cabot G, Bazant Z. Nonlocal damage theory. Journal of Engineering Mechanics 1987; 113(10): $1512-1533$.

25. Allix O, Deu J. Delayed-damage modelling for fracture prediction of laminated composites under dynamic loading. Engineering Transactions/Rozprawy Inzynierskie 1997; 45(1):29-46.

26. Rannou J, Limodin N, Réthoré J, Gravouil A, Ludwig W, Baietto-Dubourg M, Buffière J, Combescure A, Hild F, Roux S. Three dimensional experimental and numerical analysis of a fatigue crack. Computer Methods in Applied Mechanics and Engineering 2010; 199(21-22):1307-1325. 